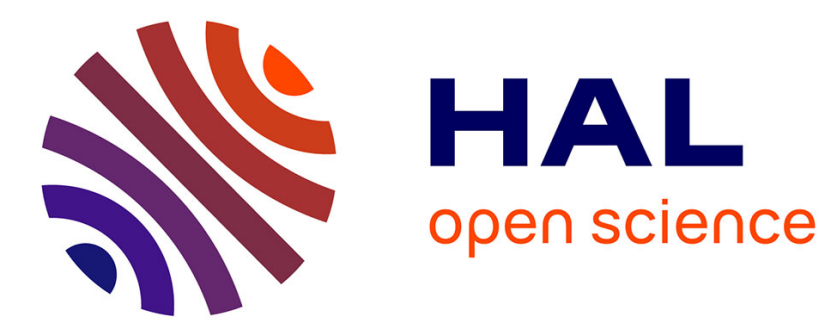

\title{
Coupled topology optimization of structure and connections for bolted mechanical systems
}

Lalaina Rakotondrainibe, Jeet Desai, Patrick Orval, Grégoire Allaire

\section{To cite this version:}

Lalaina Rakotondrainibe, Jeet Desai, Patrick Orval, Grégoire Allaire. Coupled topology optimization of structure and connections for bolted mechanical systems. 2021. hal-03273522v1

\section{HAL Id: hal-03273522 \\ https://hal.science/hal-03273522v1}

Preprint submitted on 29 Jun 2021 (v1), last revised 3 Jan 2022 (v2)

HAL is a multi-disciplinary open access archive for the deposit and dissemination of scientific research documents, whether they are published or not. The documents may come from teaching and research institutions in France or abroad, or from public or private research centers.
L'archive ouverte pluridisciplinaire HAL, est destinée au dépôt et à la diffusion de documents scientifiques de niveau recherche, publiés ou non, émanant des établissements d'enseignement et de recherche français ou étrangers, des laboratoires publics ou privés. 


\title{
Coupled topology optimization of structure and connections for bolted mechanical systems
}

\author{
L. Rakotondrainibe ${ }^{1,2} \quad$ J. Desai $^{3} \quad$ P. Orval $^{1} \quad$ G. Allaire ${ }^{2}$ \\ ${ }^{1}$ Technocentre RENAULT, 1 Avenue du Golf, 78084 Guyancourt cedex, France, \\ rakotondrainibe.anja@gmail.com, patrick.orval@renault.com \\ ${ }^{2}$ Centre de Mathématiques Appliquées, Ecole Polytechnique, Route de Saclay, 91128 Palaiseau, France, \\ gregoire.allaire@polytechnique.fr \\ ${ }^{3}$ IRT SystemX, 8, Avenue de la Vauve, 91127 Palaiseau, France, \\ jeet.desai@irt-systemx.fr
}

June 29, 2021

\begin{abstract}
This work introduces a new coupled topology optimization approach for a structural assembly. Considering several parts connected by bolts, the shape and topology of potentially each part, as well as the position and number of bolts are simultaneously optimized. The main ingredients of our optimization approach are the level-set method for structural optimization, a new notion of topological derivative of an idealized model of bolt in order to decide where it is advantageous to add a new bolt, coupled with a parametric gradient-based algorithm for its position optimization. Both idealized bolt and its topological derivative handle prestressed state complexity. Several 3d numerical test cases are performed to demonstrate the efficiency of the proposed strategy for mass minimization, considering Von Mises and fatigue constraints for the bolts and compliance constraint for the structure. In particular, a simplified but industrially representative example of an accessories bracket for car engines demonstrates significant benefits. Optimizing both the structure and its connections reduces the mass by $24 \%$ compared to classical "structure-only" optimization.
\end{abstract}

Keywords: Topology optimization, Assembled system, Bolt, Topological derivative, Level-set method 


\section{Contents}

1 Introduction $\quad 3$

2 Mechanical framework for a bolted assembly $\quad 4$

2.1 Idealized model of a bolt . . . . . . . . . . . . . . . . . . . . . . . . . . 4

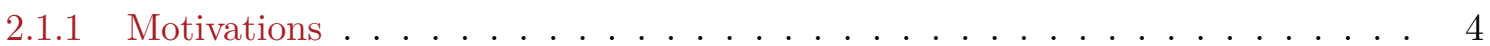

2.1.2 Analytic constitutive behavior . . . . . . . . . . . . . . 4

2.2 Generic assembly . . . . . . . . . . . . . . . . . . . . . . 5

2.3 Contact model . . . . . . . . . . . . . . . . . . . . . . . 6

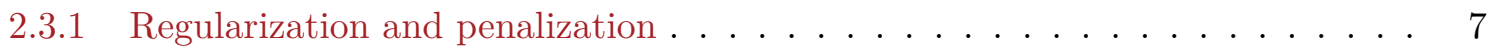

2.3 .2 Adjoint state . . . . . . . . . . . . . . . . . 8

2.4 Elasticity problems . . . . . . . . . . . . . . . . . . . 9

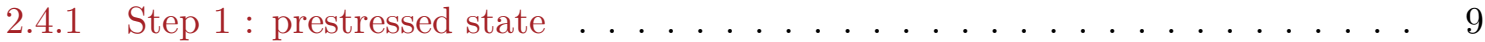

2.4 .2 Step 2 : in-service state . . . . . . . . . . . . . . . . 10

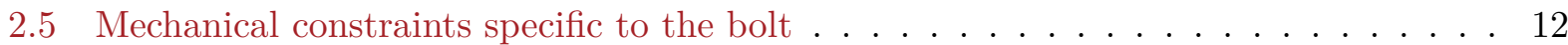

2.5.1 Torsor of inner efforts . . . . . . . . . . . . . . . . . 12

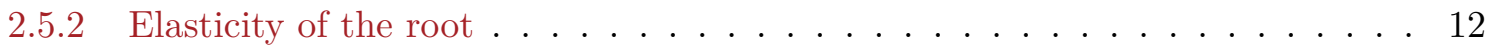

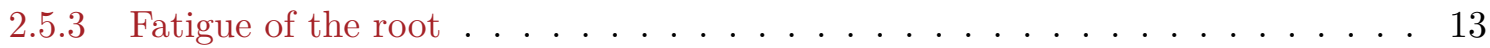

3 Optimization methods $\quad 13$

3.1 Shape optimization . . . . . . . . . . . . . . . . . . . 13

3.1 .1 Level-set method . . . . . . . . . . . . . . . . . . . . . . 13

3.1.2 Hadamard's boundary variation method . . . . . . . . . . . . . . . 14

3.2 Bolt location optimization . . . . . . . . . . . . . . . . . . . 14

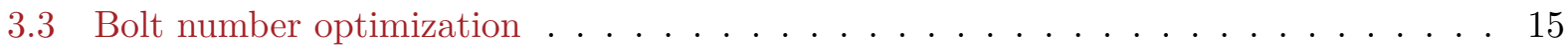

4 Numerical illustrations $\quad 16$

4.1 Academic use case . . . . . . . . . . . . . . . . . . . 16

4.1 .1 Setting of the problem . . . . . . . . . . . . . . . . 16

4.1.2 Test of the topological derivative . . . . . . . . . . . . . . . 17

4.1.3 Structure optimization with a fixed bolt . . . . . . . . . . . . . . 17

4.1.4 Coupled optimization of structure and bolt location . . . . . . . . . 18

4.2 Simplified industrial use case . . . . . . . . . . . . . . . . 20

4.2 .1 Setting of the problem . . . . . . . . . . . . . . . 20

4.2 .2 Optimization problem . . . . . . . . . . . . . . . . . 22

4.2 .3 Reference optimization: structure alone . . . . . . . . . . . . . . . 24

4.2 .4 Coupled optimization . . . . . . . . . . . . . . . . . 24

5 Conclusion and perspectives $\quad 29$ 


\section{Introduction}

Design of mechanical parts in automotive industry now benefits from topology optimization, mostly in a linear mechanics context. Latest improvements in topology optimization result in shapes that are easier to interpret. Moreover, significant efforts have been made to reduce the computational burden and to incorporate non-linear mechanics. Yet the scope of existing research mainly focuses on the optimization of a single part. Important industrial applications involve modeling of mechanical connections between two or more disjointed parts. Incorporating these connections as design variables in the topology optimization process should greatly benefit to weight reduction.

Indeed, former works using classical topology optimization techniques have proved their efficiency. However, they are limited to springs and supports (elastic or rigid), whereas connections involved in solid parts assemblies are mostly bolts or screws. The level-set method is employed in [31, 45] to optimize both structure and supports. In [7, 50], authors use a density approach, such as SIMP, to find the optimal supports configuration. Another example of supports modeled by elastic springs is investigated in [4]. The optimal locations and number of springs that support a vibrating cantilever beam are optimized to minimize the shear force applied to the beam. In [40], support locations are optimized with a gradient-based method to solve a frequency optimization problem. In [43], connections are successively modeled by elastic then by rigid supports in order to maximize the fundamental frequency of a beam or plate structure through the supports location optimization. Classical shape optimization techniques, supplemented by non-overlapping constraints between parts, provide satisfactory results for the optimization of supports in [51].

Technological implementation of tightening process involves complexity in terms of mechanical behavior, not only for bolts as individual components (their own resistance) but also for the entire system (mechanical response considering prestressed state). In [18], spot-welds and adhesive bonds are modeled by springs scaled by a penalization parameter. A multi-point constraints (MPC) based method is applied in [48, 49] to define rivets or bolts connections in the design of multicomponent systems. In [17], bolts are modeled by bar elements connecting two circular surfaces embodying fastener holes to minimize the structure compliance under fatigue failure constraints with Sines criterion [35]. An optimal layout of fasteners is determined in [26] with a fatigue design. Besides, evolutionary and genetic algorithms are proposed in [21, 41, 42] to optimize structure and connections.

More synthetically, recent works allow to express limitations of available methods for connections pattern optimization: SIMP topology optimization is efficient but fails to deal with non-linearity and still requires human ingredients to achieve discretized patterns for connections, combinatorial analysis of parametrized models in conjunction with metamodels can handle non-linearity but may miss the true optimal pattern for connections when cumulated computational time is bounded $[33,46]$. This research work in topology optimization takes on this double challenge: to consider the mechanical behavior complexity of bolted systems, and to solve what looks like a combinatorial problem with regards to connections pattern. Furthermore, as topology relies on identification and adjustment to load paths, a high precision model for the behavior of a bolt (see e.g. [6, 24, 47, 52]) would be computationally expensive and useless for the optimization of large assemblies. It is worth simplifying the model of bolt connection in some cases (computational limitations, complex mechanical behavior) [5, 13] and even considering analytical models [9, 14].

This paper investigates a coupled optimization of mechanical parts and their bolted connections and is organized as follows. In Section 2, we define a model of assembly using an idealized long bolt and a sliding contact formulation. The prestress induced by the tightening process is established with mechanically and mathematically rigorous justifications. Mechanical design constraints dependent on bolt strength and resistance are derived for optimization needs. Section 3 presents the methods used to optimize concurrently the structure, and the location and number of bolts. The structure is optimized using the level-set method combined with the Hadamard's boundary variation method $[3,44]$. Bolts location is optimized using a parametric gradient-based algorithm. Bolts number is 
optimized using the concept of topological derivative $[8,38]$ to add an idealized bolt at the optimal location. We use the notion developed in [32] to add a supplementary bolt in the assembly. Finally, Section 4 numerically illustrates the resulting coupled optimization, applied first to an academic test case, and then to a simplified, but representative, industrial test case.

\section{Mechanical framework for a bolted assembly}

\subsection{Idealized model of a bolt}

\subsubsection{Motivations}

A representative mechanical behavior between two bolted parts demands a model of bolt connection to include its whole technical implementation. However, fine modeling of the bolt by the Finite Element (FE) method is not necessary if one is not interested in local results, which is the case for topology optimization of solid parts. In the case of long bolts, it may be advantageous to consider an analytical model which is established in this section. Physical representativeness for load paths is kept at first order using this idealized model. Moreover, this analytical model is computationally cheap, easy to plug-in in an optimization loop and amenable to the notion of topological derivative.

\subsubsection{Analytic constitutive behavior}

We propose a simple representation in which the bolt is represented by two spheres symbolizing its head and its threads, respectively denoted by $\omega_{A}$ and $\omega_{B}$ (see Fig. 1). These spheres allow efforts transmission and displacements continuity between the bolt extremities and the jointed parts. Their centers are separated by a distance $\ell$, in the direction of a unit vector $\boldsymbol{e}$ oriented from $\omega_{A}$ to $\omega_{B}$, and have a radius $r_{b}$. The length $\ell$ stands for the effective implantation length of the bolt in the assembly and the radius $r_{b}$ corresponds to the effective radius of the bolt $[14,15]$.

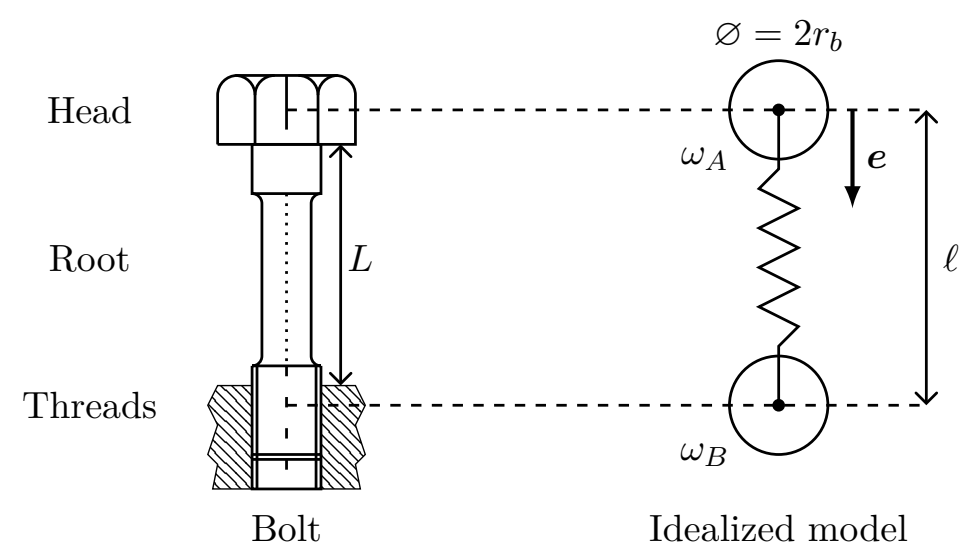

Figure 1: Long bolt and the idealized model

Remark 1. According to [14, 15], the effective lenght $\ell$ is approximated from the implantation length $L$ by $\ell=L+1.6 r_{b}$.

The idealized model is associated to the rigidity matrix, $\mathrm{K}_{b}$, obeying to the Euler-Bernoulli condition for long beam which may read $\ell \geq 10 r_{b}$. The study is carried out in the framework of 
small displacements. Thus, the rigidity matrix reads at first order

$$
\mathrm{K}_{b}=\frac{E_{b} \pi r_{b}^{2}}{\ell}\left(\begin{array}{ccc:ccc}
1 & 0 & 0 & 0 & 0 & 0 \\
0 & 3\left(\frac{r_{b}}{\ell}\right)^{2} & 0 & 0 & 0 & -\frac{3}{2} \frac{r_{b}^{2}}{\ell} \\
0 & 0 & 3\left(\frac{r_{b}}{\ell}\right)^{2} & 0 & \frac{3}{2} \frac{r_{b}^{2}}{\ell} & 0 \\
\hdashline 0 & 0 & 0 & \frac{r_{b}^{2}}{4(1+\nu)} & 0 & 0 \\
0 & 0 & \frac{3}{2} \frac{r_{b}^{2}}{\ell} & 0 & r_{b}^{2} & 0 \\
0 & -\frac{3}{2} \frac{r_{b}^{2}}{\ell} & 0 & 0 & 0 & r_{b}^{2}
\end{array}\right) \text {, }
$$

where $E_{b}$ is the Young modulus and $\nu$ is Poisson's ratio of the bolt. The subscript "b" indicates that the matrix is written in the local orthonormal cylindrical coordinates system of the bolt where unit vector $\boldsymbol{e}$ defines the first axis. The expression of the matrix $\mathrm{K}_{b}(1)$ is obtained with an expansion at first order considering Euler-Bernoulli condition for long beam. Both spheres are represented in the $\mathrm{FE}$ model and the action of the root is modeled as a remote interaction between them, using an analytical 6 degrees of freedom (DOFs) linear-spring law

$$
\boldsymbol{F}_{b}=\mathrm{K}_{b} \boldsymbol{L}_{b}
$$

with $\boldsymbol{F}_{b}$ the generalized forces and $\boldsymbol{L}_{b}$ the generalized lengthenings of the bolt in the local coordinate system. The first three components of $\boldsymbol{L}_{b}$ stand for relative translations between $\omega_{A}$ and $\omega_{B}$ and the last three for relative rotations.

The rigidity matrix $\mathrm{K}_{b}$ is decomposed into a traction/torsion matrix and a shear/flexion matrix, respectively denoted $\overline{\mathrm{K}}_{b}$ and $\tilde{\mathrm{K}}_{b}$. They read

$$
\begin{gathered}
\overline{\mathrm{K}}_{b}=\frac{E_{b} \pi r_{b}^{2}}{\ell}\left(\begin{array}{ccc:cccc}
1 & 0 & 0 & 0 & 0 & 0 \\
0 & 0 & 0 & 0 & 0 & 0 \\
0 & 0 & 0 & 0 & 0 & 0 \\
\hdashline 0 & 0 & 0 & \frac{r_{b}^{2}}{4(1+\nu)} & 0 & 0 \\
0 & 0 & 0 & 0 & 0 & 0 \\
0 & 0 & 0 & 0 & 0 & 0
\end{array}\right) \\
\tilde{\mathrm{K}}_{b}=\frac{E_{b} \pi r_{b}^{2}}{\ell}\left(\begin{array}{ccc:ccc}
0 & 0 & 0 & 0 & 0 & 0 \\
0 & 3\left(\frac{r_{b}}{\ell}\right)^{2} & 0 & 0 & 0 & -\frac{3}{2} \frac{r_{b}^{2}}{\ell} \\
0 & 0 & 3\left(\frac{r_{b}}{\ell}\right)^{2} & 0 & \frac{3}{2} \frac{r_{b}^{2}}{\ell} & 0 \\
\hdashline 0 & 0 & 0 & 0 & 0 & 0 \\
0 & 0 & \frac{3}{2} \frac{r_{b}^{2}}{\ell} & 0 & r_{b}^{2} & 0 \\
0 & -\frac{3}{2} \frac{r_{b}^{2}}{\ell} & 0 & 0 & 0 & r_{b}^{2}
\end{array}\right) .
\end{gathered}
$$

We remove the subscript "b" when the matrices are written in the global Cartesian coordinates.

\subsection{Generic assembly}

Let us consider two disjointed parts $\Omega_{A}$ and $\Omega_{B}$ connected by one bolt (see Fig. 2). Let $\Omega$ be the union of $\Omega_{A}$ and $\Omega_{B}$. This union is assumed to be a smooth bounded domain of $\mathbb{R}^{3}$. The boundary of this domain is composed of three disjointed parts, $\partial \Omega=\Gamma \cup \Gamma_{N} \cup \Gamma_{D}$. Traction-free, Neumann and Dirichlet boundary conditions are respectively imposed on $\Gamma, \Gamma_{N}$ and $\Gamma_{D}$. The study is carried 
out in the framework of small deformations. For a displacement field $\boldsymbol{u}$, the strain tensor is defined by $\varepsilon(\boldsymbol{u})=\frac{1}{2}\left(\nabla \boldsymbol{u}+\nabla^{T} \boldsymbol{u}\right)$. Each part $\Omega_{A}\left(\right.$ resp. $\left.\Omega_{B}\right)$ is filled with a linear isotropic elastic material, with Lamé coefficients $\mu_{A}$ and $\lambda_{A}$ (resp. $\mu_{B}$ and $\lambda_{B}$ ). The stress tensor is then given by the Hooke's law

$$
\mathbf{C} \varepsilon(\boldsymbol{u})= \begin{cases}2 \mu_{A} \varepsilon(\boldsymbol{u})+\lambda_{A} t r(\varepsilon(\boldsymbol{u})) \mathbf{I} & \text { in } \Omega_{A} \\ 2 \mu_{B} \varepsilon(\boldsymbol{u})+\lambda_{B} \operatorname{tr}(\varepsilon(\boldsymbol{u})) \mathbf{I} & \text { in } \Omega_{B}\end{cases}
$$

Several interface models on $\mathrm{S}$ can be considered. For example, a perfect interface is used in [32]. In this paper, we consider the contact model described in Section 2.3.

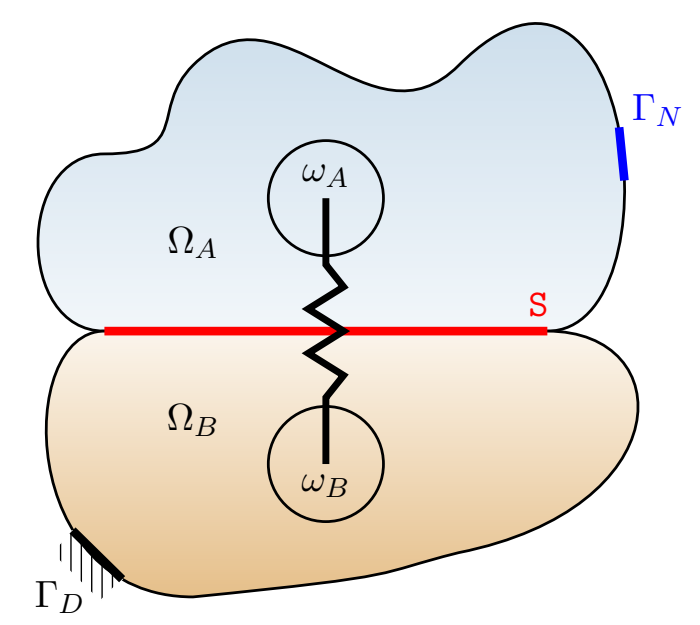

Figure 2: Assembled system with one bolt

\subsection{Contact model}

We now describe the contact model used in this work, which is frictionless. As shown in Fig. 2, let $\Omega_{A}$ and $\Omega_{B}$ be two solids in contact at the interface $\mathrm{S}$. The interface $\mathrm{S}$ is also denoted $\mathrm{S}^{+}$when it is seen as a subset of the boundary of $\Omega_{A}$ and $\mathrm{S}^{-}$when it is a subset of the boundary of $\Omega_{B}$. Let $\boldsymbol{n}^{+}$ and $\boldsymbol{n}^{-}$be the outward normal to $\mathrm{S}^{+}$and $\mathrm{S}^{-}$respectively. The value of $\boldsymbol{u}$ on $\mathrm{S}^{+}$and $\mathrm{S}^{-}$shall be denoted by $\boldsymbol{u}^{+}$and $\boldsymbol{u}^{-}$respectively. Let the jump in displacement be $[\boldsymbol{u}]=\boldsymbol{u}^{-}-\boldsymbol{u}^{+}$. For the solids $\Omega_{A}$ and $\Omega_{B}$, the Signorini contact boundary conditions are formulated assuming

- Absence of penetration:

$$
[\boldsymbol{u}] \cdot \boldsymbol{n}^{-} \leq 0
$$

- Contact reaction force being compressive only:

$$
\mathrm{C} \varepsilon\left(\boldsymbol{u}_{\mid \mathrm{S}^{-}}\right) \boldsymbol{n}^{-} \cdot \boldsymbol{n}^{-}=\mathrm{C} \varepsilon\left(\boldsymbol{u}_{\mid \mathrm{S}^{+}}\right) \boldsymbol{n}^{+} \cdot \boldsymbol{n}^{+} \leq 0
$$

- Complementary relation between contact and contact-force:

$$
[\boldsymbol{u}] \cdot \boldsymbol{n}^{-}\left(\mathrm{C} \varepsilon\left(\boldsymbol{u}_{\mid \mathrm{S}^{-}}\right) \boldsymbol{n}^{-} \cdot \boldsymbol{n}^{-}\right)=0 .
$$

The complementary relation implies that either there is no contact $[\boldsymbol{u}] \cdot \boldsymbol{n}^{-}<0$ and a zero contact force $C \varepsilon\left(\boldsymbol{u}_{\mid S^{-}}\right) n^{-} \cdot \boldsymbol{n}^{-}=0$, or there is contact $[\boldsymbol{u}] \cdot \boldsymbol{n}^{-}=0$ and the contact force is non-zero. These 
contact boundary conditions (5)-(7) ought to be satisfied with the momentum balance equation

$$
\left\{\begin{aligned}
\operatorname{div}(\mathrm{C} \varepsilon(\boldsymbol{u})) & =\mathbf{0} & & \text { in } \Omega_{A} \text { and in } \Omega_{B} \\
\mathrm{C} \varepsilon(\boldsymbol{u}) \cdot \boldsymbol{n} & =\boldsymbol{g} & & \text { on } \Gamma_{N} \\
\mathrm{C} \varepsilon(\boldsymbol{u}) \cdot \boldsymbol{n} & =\mathbf{0} & & \text { on } \Gamma \\
\boldsymbol{u} & =\mathbf{0} & & \text { on } \Gamma_{D},
\end{aligned}\right.
$$

where $\boldsymbol{n}$ denotes the outward normal to $\Omega_{A}$ and $\Omega_{B}$, and $\boldsymbol{g}$ denotes an external surface load.

We introduce the space of displacements $\boldsymbol{u}$ as

$$
V=\left\{\boldsymbol{u} \in\left(H^{1}\left(\Omega_{A}\right) \cap H^{1}\left(\Omega_{B}\right)\right)^{3}: \boldsymbol{u}=0 \text { on } \Gamma_{D}\right\}
$$

and the set $K$ of displacements satisfying the non penetration condition on $\mathrm{S}$ as

$$
K=\left\{\boldsymbol{u} \in V: \quad[\boldsymbol{u}] \cdot \boldsymbol{n}^{-} \leq 0, \text { on } \mathrm{S}\right\} .
$$

It is easily seen that $K$ is a convex subset of $V$. We introduce a bilinear form $a: V \times V \mapsto \mathbb{R}$

$$
a(\boldsymbol{u}, \boldsymbol{v})=\int_{\Omega} \mathrm{C} \varepsilon(\boldsymbol{u}): \varepsilon(\boldsymbol{v}) d V .
$$

Only if we assume that the dirichlet boundary $\Gamma_{D}$ is present on both the boundaries $\partial \Omega_{A}$ and $\partial \Omega_{B}$, we can show that the bilinear form $a(\cdot, \cdot)$ is coercive in $V$. We then introduce a linear form $l: V^{*} \mapsto \mathbb{R}$

$$
l(\boldsymbol{u})=\int_{\Gamma_{N}} \boldsymbol{g} \cdot \boldsymbol{u} d S,
$$

where $\boldsymbol{g} \in\left(L^{2}\left(\Gamma_{N}\right)\right)^{3}$ is the external force. To determine the weak form of equations (8) with its boundary conditions, consider a test function $\boldsymbol{v} \in K$, multiply (8) by $\boldsymbol{v}-\boldsymbol{u}$ and integrate by parts over $\Omega_{A}$ and $\Omega_{B}$, resulting in

$$
\begin{aligned}
\int_{\Omega} \mathrm{C} \varepsilon(\boldsymbol{u}) & : \varepsilon(\boldsymbol{v}-\boldsymbol{u}) d V \\
& =\int_{\mathrm{S}^{+}} \mathrm{C} \varepsilon\left(\boldsymbol{u}^{+}\right) \cdot \boldsymbol{n}^{+}\left(\boldsymbol{v}^{+}-\boldsymbol{u}^{+}\right) d S+\int_{\mathrm{S}^{-}} \mathrm{C} \varepsilon\left(\boldsymbol{u}^{-}\right) \cdot \boldsymbol{n}^{-}\left(\boldsymbol{v}^{+}-\boldsymbol{u}^{-}\right) d S+\int_{\Gamma_{N}} \boldsymbol{g} \cdot(\boldsymbol{v}-\boldsymbol{u}) d S
\end{aligned}
$$

Then, injecting the contact boundary conditions (5)-(7), one ends up with a weak form that is an inequality. Using the bilinear form $a(\cdot, \cdot)$ and linear form $l(\cdot)$ defined in (9) and (10), this inequality reads: find $\boldsymbol{u} \in K$ such that

$$
a(\boldsymbol{u}, \boldsymbol{v}-\boldsymbol{u}) \geq l(\boldsymbol{v}-\boldsymbol{u}) \quad \forall \boldsymbol{v} \in K .
$$

The variational inequality (12) admits a unique solution $\boldsymbol{u} \in K[20]$.

\subsubsection{Regularization and penalization}

In order to determine the shape derivative for the contact problem (12), it ought to be differentiated with respect to $\boldsymbol{u}$. Since there is a constraint on $\boldsymbol{u} \in K$, where $K$ is a convex set, the notion of classical differentiation is lost and one needs to resort to the notion of conical derivative [23]. This has been performed in the context of the static contact problem with and without friction in [37]. The conical derivative so obtained is hard to implement numerically.

In order to simplify the differentiation and numerical implementation of the derivative, we replace the constrained problem (12), where the solution and the test function must belong to the convex set $K$, by a penalized version, where the constraint $\boldsymbol{u} \in K$ is penalized. The penalization 
method in the context of contact mechanics has been elaborated in [11]. The idea of the penalization method is to search for a solution in $V$ by penalizing the constraint (5), thereby forcing the solution to belong to $K$ in the limit of a vanishing penalization parameter. The penalization of the contact boundary condition converts the inequality (12) into an equality. We introduce a penalization parameter $0<\epsilon \ll 1$ and define a function $j_{\epsilon}: V \mapsto \mathbb{R}$

$$
j_{\epsilon}(\boldsymbol{u})=\frac{1}{\epsilon} \int_{S} \int_{0}^{[\boldsymbol{u}] \cdot \boldsymbol{n}} M_{\epsilon}(t) d t d s
$$

where $M_{\epsilon}(\cdot)$ is the regularization of the $\max (\cdot, 0)$ function given by

$$
M_{\epsilon}(t)=\frac{1}{2}\left(t+\sqrt{t^{2}+\epsilon^{2}}\right) .
$$

The term $j_{\epsilon}(\boldsymbol{u})$ penalizes the constraint (5). Using the above, the solution to (12) $\boldsymbol{u}$ is then approximated by $\boldsymbol{u}_{\epsilon}$, defined as the unique minimizer in $V$ of

$$
\min _{\boldsymbol{v} \in V}\left(\frac{1}{2} a(\boldsymbol{v}, \boldsymbol{v})-l(\boldsymbol{v})+j_{\epsilon}(\boldsymbol{v})\right)
$$

where $j_{\epsilon}(\cdot)$, defined in (13), is convex and thus ensures the existence and uniqueness of the solution. Since the functional $j_{\epsilon}(\cdot)$ is smooth, the minimization (15) is equivalent to Euler-Lagrange optimality condition, which reads: find $\boldsymbol{u}_{\epsilon} \in V$ such that, for any $\boldsymbol{v} \in V$,

$$
a\left(\boldsymbol{u}_{\epsilon}, \boldsymbol{v}\right)-l(\boldsymbol{v})+\left\langle j_{\epsilon}^{\prime}\left(\boldsymbol{u}_{\epsilon}\right), \boldsymbol{v}\right\rangle=0,
$$

where the pairing $\langle\cdot, \cdot\rangle$ denotes the scalar product on $L^{2}(\Omega)^{3}$,

$$
\langle\boldsymbol{u}, \boldsymbol{v}\rangle=\int_{\Omega} \boldsymbol{u} \cdot \boldsymbol{v} d V \quad \forall \boldsymbol{u}, \boldsymbol{v} \in L^{2}(\Omega)^{3} .
$$

We shall be treating the above contact formulation for the rest of the article.

\subsubsection{Adjoint state}

We now briefly talk about the shape optimization problem for (16). Given a functional

$$
J(\Omega)=\int_{\Omega} m\left(\boldsymbol{u}_{\epsilon}(\Omega)\right) d V+\int_{\Gamma_{N}} q\left(\boldsymbol{u}_{\epsilon}(\Omega)\right) d S,
$$

where $\boldsymbol{u}_{\epsilon}(\Omega)$ is the solution to (16), we wish to determine $\Omega$ such that

$$
\Omega=\underset{\bar{\Omega} \in \mathcal{U}_{a d}}{\arg \min } J(\bar{\Omega}),
$$

where $\mathcal{U}_{a d}$ is the space of admissible shapes. The adjoint state $\boldsymbol{p}_{\epsilon}$ for $J(\Omega)$ solves the following equations

$$
\left\{\begin{aligned}
\operatorname{div}\left(\mathrm{C} \varepsilon\left(\boldsymbol{p}_{\epsilon}\right)\right) & =m^{\prime}\left(\boldsymbol{u}_{\epsilon}\right) & & \text { in } \Omega_{A} \text { and in } \Omega_{B}, \\
\mathrm{C} \varepsilon\left(\boldsymbol{p}_{\epsilon}\right) \cdot \boldsymbol{n}^{-} & =-\frac{1}{\epsilon} M_{\epsilon}^{\prime}\left(\left[\boldsymbol{u}_{\epsilon}\right] \cdot \boldsymbol{n}^{-}\right)\left[\boldsymbol{p}_{\epsilon}\right] \cdot \boldsymbol{n}^{-} & & \text {on } \mathrm{S}^{-}, \\
\mathrm{C} \varepsilon\left(\boldsymbol{p}_{\epsilon}\right) \cdot \boldsymbol{n}^{+} & =-\frac{1}{\epsilon} M_{\epsilon}^{\prime}\left(\left[\boldsymbol{u}_{\epsilon}\right] \cdot \boldsymbol{n}^{-}\right)\left[\boldsymbol{p}_{\epsilon}\right] \cdot \boldsymbol{n}^{-} & & \text {on } \mathrm{S}^{+}, \\
\mathrm{C} \varepsilon\left(\boldsymbol{p}_{\epsilon}\right) \cdot \boldsymbol{n} & =-q^{\prime}\left(\boldsymbol{u}_{\epsilon}\right) & & \text { on } \Gamma_{N}, \\
\mathrm{C} \varepsilon\left(\boldsymbol{p}_{\epsilon}\right) \cdot \boldsymbol{n} & =\mathbf{0} & & \text { on } \Gamma, \\
\boldsymbol{u} & =\mathbf{0} & & \text { on } \Gamma_{D} .
\end{aligned}\right.
$$

The derivation of the above adjoint equation has been performed in [22]. The weak form of (17) reads: find $\boldsymbol{p}_{\epsilon} \in V$ such that

$$
a\left(\boldsymbol{\psi}, \boldsymbol{p}_{\epsilon}\right)+\frac{1}{\epsilon} \int_{\mathrm{S}}[\boldsymbol{\psi}] \cdot \boldsymbol{n}^{-} M_{\epsilon}^{\prime}\left(\left[\boldsymbol{u}_{\epsilon}\right] \cdot \boldsymbol{n}^{-}\right)\left[\boldsymbol{p}_{\epsilon}\right] \cdot \boldsymbol{n}^{-} d S=-\int_{\Omega} m^{\prime}\left(\boldsymbol{u}_{\epsilon}\right) \boldsymbol{\varphi} d V-\int_{\Gamma_{N}} q^{\prime}\left(\boldsymbol{u}_{\epsilon}\right) \boldsymbol{\varphi} d S \quad \forall \boldsymbol{\psi} \in V
$$




\subsection{Elasticity problems}

This section sets up elasticity problems with an idealized bolt, including the contact model described in Section 2.3. In the following, we assume that the lengthening of the root is the difference between the average degrees of freedom in $\omega_{A}$ and $\omega_{B}$. Let us introduce the notation $\boldsymbol{W}^{A}$, for the average degrees of freedom on $\omega_{A}$ of an arbitrary displacement field $\boldsymbol{w}$

$$
\boldsymbol{W}^{A}=\left(\begin{array}{c}
f \boldsymbol{w} d V \\
\frac{1}{\omega_{A}} f_{\omega_{A}} \operatorname{curl}(\boldsymbol{w}) d V
\end{array}\right)=\left(\begin{array}{c}
\frac{1}{\left|\omega_{A}\right|} \int_{\omega_{A}} \boldsymbol{w} d V \\
\frac{1}{2\left|\omega_{A}\right|} \int_{\omega_{A}} \operatorname{curl}(\boldsymbol{w}) d V
\end{array}\right),
$$

and likewise for $\boldsymbol{W}^{B}$ in $\omega_{B}$.

The idealized model is described through Euler-Bernoulli theory which relies on the displacement of the neutral fiber of the bolt. It leads to the rigidification of the extremities of the bolt which is strictly considered here with rigid body motions in the head and the threads of the idealized bolt. The space of admissible displacements then corresponds to zero displacement on $\Gamma_{D}$ and rigid body motions in $\omega_{A}$ and $\omega_{B}$

$$
\mathcal{W}=\left\{\begin{array}{l}
\boldsymbol{w} \in\left(H^{1}\left(\Omega_{A}\right) \cap H^{1}\left(\Omega_{B}\right)\right)^{3}, \boldsymbol{w}=\mathbf{0} \text { on } \Gamma_{D}, \\
\forall \boldsymbol{x} \in \omega_{A} \boldsymbol{w}(\boldsymbol{x})=\mathcal{C}_{a}+\mathrm{R}_{a} \boldsymbol{x}, \\
\forall \boldsymbol{x} \in \omega_{B} \boldsymbol{w}(\boldsymbol{x})=\mathcal{C}_{b}+\mathrm{R}_{b} \boldsymbol{x}
\end{array}\right\},
$$

where $\left(\mathcal{C}_{a}, \mathcal{C}_{b}\right) \in \mathbb{R}^{3} \times \mathbb{R}^{3}$ are translations and $\mathrm{R}_{a}=-\mathrm{R}_{a}^{T}$ and $\mathrm{R}_{b}=-\mathrm{R}_{b}^{T}$ are anti-symmetric $3 \times 3$ matrices modeling infinitesimal rotations.

Remark 2. In numerical applications of Section 4, rigid body motions are applied by penalization of material properties.

\subsubsection{Step 1 : prestressed state}

Correct mechanical modeling for bolted assembly requires to consider a two-step history problem: firstly, tightening process leading to a prestressed state or "Step 1", then in-service conditions leading to in-service state or "Step 2". In practice, a bolted joint is carried out by applying a tightening torque to the head of the bolt. It creates traction and torsion of the bolt root and local compression of the jointed parts. Forgetting inner tightening torsion in the following, the prestress is obtained by applying a given external tensile force between both extremities of the root (see Fig. 3), regardless of axial and torsionnal elongations of the root. Euler-Bernoulli slenderness and elasticity allow this approximation, exactly as proposed in computational softwares, such as ABAQUS [36] and NX NASTRAN [1].

Thus, this first step consists in identifying the extremities $\omega_{A}$ and $\omega_{B}$ in the domain $\Omega$ and having the root behave according to the rigidity matrix $\tilde{\mathrm{K}}$ (i.e. only in shear/flexion modes). The prestress external force of amplitude $Q>0$ is remotely applied between each domain $\omega_{A}$ and $\omega_{B}$ along the direction $\boldsymbol{e}$. This elasticity problem, including contact, results in a displacement field $\boldsymbol{u}_{S 1}$ computed over the domain $\Omega$. The subscript "S1" refers to Step 1. 


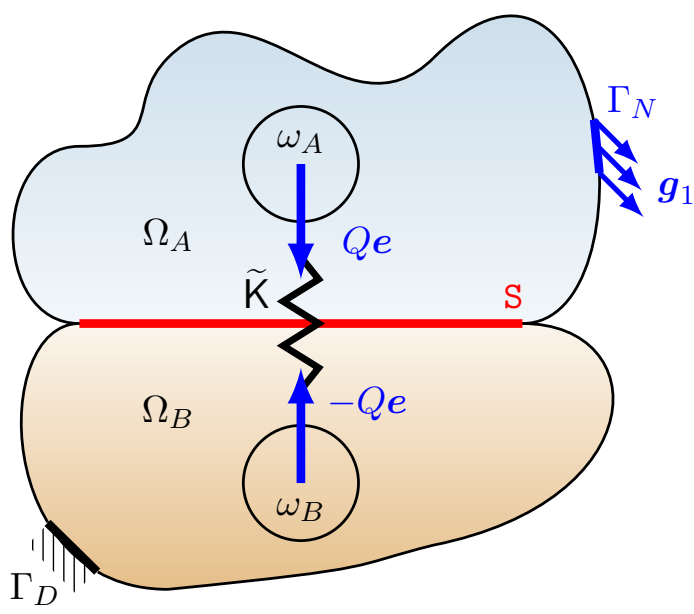

Figure 3: Step 1

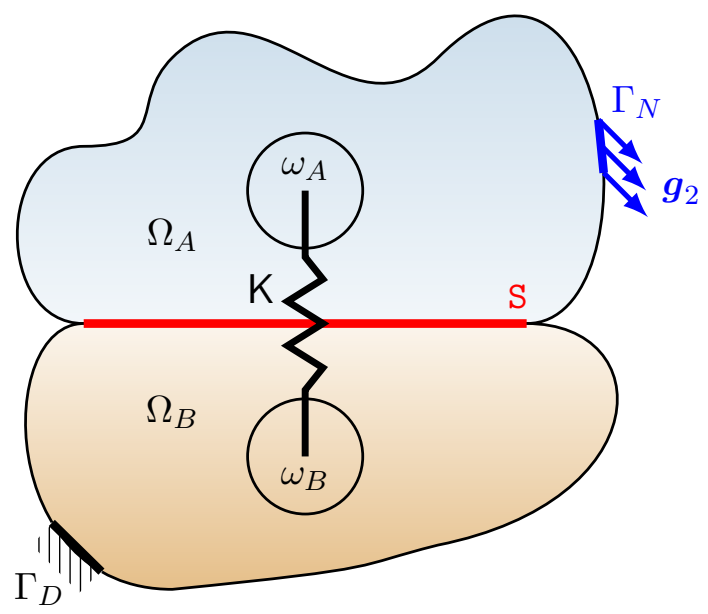

Figure 4: Step 2

Let $E_{S 1}$ be the energy functional

$$
\begin{aligned}
E_{S 1}(\boldsymbol{w})= & \frac{1}{2} \int_{\Omega \backslash\left(\omega_{A} \cup \omega_{B}\right)} C \varepsilon(\boldsymbol{w}): \varepsilon(\boldsymbol{w}) d V-\int_{\Omega} \boldsymbol{f}_{1} \cdot \boldsymbol{w} d V-\int_{\Gamma_{N}} \boldsymbol{g}_{1} \cdot \boldsymbol{w} d S \\
& +\frac{1}{2}\left(\boldsymbol{W}^{B}-\boldsymbol{W}^{A}\right)^{T} \tilde{\mathrm{K}}\left(\boldsymbol{W}^{B}-\boldsymbol{W}^{A}\right)-Q \boldsymbol{f}_{\omega_{A}} \boldsymbol{w} \cdot \boldsymbol{e} d V-(-Q) f_{\omega_{B}} \boldsymbol{w} \cdot \boldsymbol{e} d V \\
& +\frac{1}{\epsilon} \int_{\mathrm{S}} \int_{0}^{[\boldsymbol{w}] \cdot \boldsymbol{n}} M_{\epsilon}(t) d t d S .
\end{aligned}
$$

The first term is the elastic strain energy. Rigid body motions in each sphere implies that $\omega_{A}$ and $\omega_{B}$ can be excluded from the elastic energy computation. Body and surface holding forces $\boldsymbol{f}_{1}$ and $\boldsymbol{g}_{1}$ are applied to the assembly. The fourth term is the energy of the root subjected to shear and flexion solicitations. Finally, the prestress force of amplitude $Q$ is applied. The minimum potential energy principle then states that the displacement field describing the system in the first step is the unique minimizer $\boldsymbol{u}_{S 1}$ of (19) in $\mathcal{W}$, i.e.,

$$
E_{S 1}\left(\boldsymbol{u}_{S 1}\right)=\min _{\boldsymbol{w} \in \mathcal{W}} E_{S 1}(\boldsymbol{w})
$$

It leads to the following variational problem :

Find $\boldsymbol{u}_{S 1} \in \mathcal{W}$ s.t. $\forall \boldsymbol{w} \in \mathcal{W}, \int_{\Omega \backslash\left(\omega_{A} \cup \omega_{B}\right)} C \varepsilon\left(\boldsymbol{u}_{S 1}\right): \varepsilon(\boldsymbol{w}) d V-\int_{\Omega} \boldsymbol{f}_{1} \cdot \boldsymbol{w} d V-\int_{\Gamma_{N}} \boldsymbol{g}_{1} \cdot \boldsymbol{w} d S$

$+\left(\boldsymbol{U}_{S 1}^{B}-\boldsymbol{U}_{S 1}^{A}\right)^{T} \tilde{\mathrm{K}}\left(\boldsymbol{W}^{B}-\boldsymbol{W}^{A}\right)+Q\left(f_{\omega_{B}} \boldsymbol{w} \cdot \boldsymbol{e} d V-f_{\omega_{A}} \boldsymbol{w} \cdot \boldsymbol{e} d V\right)+\frac{1}{\epsilon} \int_{\mathrm{S}} M_{\epsilon}\left(\left[\boldsymbol{u}_{S 1}\right] \cdot \boldsymbol{n}\right)[\boldsymbol{w}] \cdot \boldsymbol{n} d S=0$.

\subsubsection{Step 2 : in-service state}

Once the first step is achieved, the second step consists in evaluating the previously stressed assembly submitted to in-service external loads $\boldsymbol{f}_{2}$ and $\boldsymbol{g}_{2}$, as shown in Fig. 4, where holding forces $\boldsymbol{f}_{1}$ and $\boldsymbol{g}_{1}$ 
have been removed. The root behaves in all 6 degrees of freedom according to its rigidity matrix $\mathrm{K}$. The displacement field $\boldsymbol{u}_{S 1}$ involves a prestressed state and is imposed as a pre-displacement field over the domain $\Omega$. In the same manner, now in the second step, the prestress force of amplitude $Q$ is acting as an internal force. This second elasticity problem results in a displacement field $\boldsymbol{u}_{S 2}$ computed over the domain $\Omega$. The subscript "S2" refers to Step 2.

Let $E_{S 2}$ be the energy functional

$$
\begin{aligned}
E_{S 2}(\boldsymbol{w})= & \frac{1}{2} \int_{\Omega \backslash\left(\omega_{A} \cup \omega_{B}\right)} \mathrm{C} \varepsilon(\boldsymbol{w}): \varepsilon(\boldsymbol{w}) d V-\int_{\Omega} \boldsymbol{f}_{2} \cdot \boldsymbol{w} d V-\int_{\Gamma_{N}} \boldsymbol{g}_{2} \cdot \boldsymbol{w} d S+\int_{\Omega} \boldsymbol{f}_{1} \cdot \boldsymbol{w} d V+\int_{\Gamma_{N}} \boldsymbol{g}_{1} \cdot \boldsymbol{w} d S \\
& +\frac{1}{2}\left(\boldsymbol{W}^{B}-\boldsymbol{W}^{A}\right)^{T} \mathrm{~K}\left(\boldsymbol{W}^{B}-\boldsymbol{W}^{A}\right) \\
& -\int_{\Omega \backslash\left(\omega_{A} \cup \omega_{B}\right)} \mathrm{C} \varepsilon\left(\boldsymbol{u}_{S 1}\right): \varepsilon(\boldsymbol{w}) d V-\left(\boldsymbol{U}_{S 1}^{B}-\boldsymbol{U}_{S 1}^{A}\right)^{T} \mathrm{~K}\left(\boldsymbol{W}^{B}-\boldsymbol{W}^{A}\right) \\
& +\frac{1}{\epsilon} \int_{S} \int_{0}^{[\boldsymbol{w}] \cdot \boldsymbol{n}} M_{\epsilon}(t) d t d S .
\end{aligned}
$$

The first term is the elastic strain energy. External in-service loads $\boldsymbol{f}_{2}$ and $\boldsymbol{g}_{2}$ are applied and the holding forces $\boldsymbol{f}_{1}$ and $\boldsymbol{g}_{1}$ are removed. The root now works according to all degrees of freedom according to the rigidity matrix $\mathrm{K}$ which appears in the sixth term. The pre-displacement field $\boldsymbol{u}_{S 1}$ acts as external forces on both parts and bolt and the corresponding energy is represented by seventh and eighth terms of $E_{S 2}$. The minimum potential energy principle then states that the displacement field describing the system in the Step 2 is the unique minimizer $\boldsymbol{u}_{S 2}$ of (22) in $\mathcal{W}$, i.e.,

$$
E_{S 2}\left(\boldsymbol{u}_{S 2}\right)=\min _{\boldsymbol{w} \in \mathcal{W}} E_{S 2}(\boldsymbol{w})
$$

It leads to the following variational problem :

$$
\begin{aligned}
& \text { Find } \boldsymbol{u}_{S 2} \in \mathcal{W} \text { s.t. } \forall \boldsymbol{w} \in \mathcal{W}, \int_{\Omega} \mathrm{C} \varepsilon\left(\boldsymbol{u}_{S 2}\right): \varepsilon(\boldsymbol{w}) d V-\int_{\Omega}\left(\boldsymbol{f}_{2}-\boldsymbol{f}_{1}\right) \cdot \boldsymbol{w} d V-\int_{\Gamma_{N}}\left(\boldsymbol{g}_{2}-\boldsymbol{g}_{1}\right) \cdot \boldsymbol{w} d S \\
& +\left(\boldsymbol{U}_{S 2}^{B}-\boldsymbol{U}_{S 2}^{A}\right)^{T} \mathrm{~K}\left(\boldsymbol{W}^{B}-\boldsymbol{W}^{A}\right)-\int_{\Omega} \mathrm{C} \varepsilon\left(\boldsymbol{u}_{S 1}\right): \varepsilon(\boldsymbol{w}) d V-\left(\boldsymbol{U}_{S 1}^{B}-\boldsymbol{U}_{S 1}^{A}\right)^{T} \mathrm{~K}\left(\boldsymbol{W}^{B}-\boldsymbol{W}^{A}\right) \\
& +\frac{1}{\epsilon} \int_{S} M_{\epsilon}\left(\left[\boldsymbol{u}_{S 2}\right] \cdot \boldsymbol{n}\right)[\boldsymbol{w}] \cdot \boldsymbol{n} d S=0 .
\end{aligned}
$$

Using the variational problem of Step 1 (21), the variational problem (24) is rewritten as

$$
\begin{aligned}
& \text { Find } \boldsymbol{u}_{S 2} \in \mathcal{W} \text { s.t. } \forall \boldsymbol{w} \in \mathcal{W}, \int_{\Omega} \mathrm{C} \varepsilon\left(\boldsymbol{u}_{S 2}\right): \varepsilon(\boldsymbol{w}) d V-\int_{\Omega} \boldsymbol{f}_{2} \cdot \boldsymbol{w} d V-\int_{\Gamma_{N}} \boldsymbol{g}_{2} \cdot \boldsymbol{w} d S \\
& +\left(\boldsymbol{U}_{S 2}^{B}-\boldsymbol{U}_{S 2}^{A}\right)^{T} \mathrm{~K}\left(\boldsymbol{W}^{B}-\boldsymbol{W}^{A}\right)-\left(\boldsymbol{U}_{S 1}^{B}-\boldsymbol{U}_{S 1}^{A}\right)^{T} \overline{\mathrm{K}}\left(\boldsymbol{W}^{B}-\boldsymbol{W}^{A}\right)+Q\left(\int_{\omega_{B}} \boldsymbol{w} \cdot \boldsymbol{e} d V-\boldsymbol{f}_{\omega_{A}} \boldsymbol{w} \cdot \boldsymbol{e} d V\right) \\
& +\frac{1}{\epsilon} \int_{\mathrm{S}} M_{\epsilon}\left(\left[\boldsymbol{u}_{S 2}\right] \cdot \boldsymbol{n}\right)[\boldsymbol{w}] \cdot \boldsymbol{n} d S=0 .
\end{aligned}
$$




\subsection{Mechanical constraints specific to the bolt}

Avoiding malfunction or failure of bolted joints requires further mechanical considerations. This section provides main technological constraints to be controlled with a meaningful physical representation $[14,15,19]$. Briefly stated, inner efforts torsor results from the root deformation and the tightening traction force $Q$. It is evaluated through the rigidity matrices and the average displacement $\boldsymbol{U}_{S 1}^{A}$ and $\boldsymbol{U}_{S 2}^{B}$ of the spheres $\omega_{A}$ and $\omega_{B}$. Technological constraints formulations are thus suitable for chain rule derivation for the purpose of topology optimization and for shape derivatives.

\subsubsection{Torsor of inner efforts}

The root inner efforts correspond to the torsor of the external efforts applied on the sphere $\omega_{B}$ by the part $\Omega_{B}$ evaluated at the center of $\omega_{B}$. It is evaluated in the local coordinate system. Denote $F^{n}$, with $1 \leq n \leq 3$, the resulting forces and $M^{n}$, with $1 \leq n \leq 3$, the resulting moments. The inner efforts at the end of the second step, in-service state, read

$$
\left(\begin{array}{c}
F^{1}\left(\boldsymbol{u}_{S 2}\right) \\
F^{2}\left(\boldsymbol{u}_{S 2}\right) \\
F^{3}\left(\boldsymbol{u}_{S 2}\right) \\
M^{1}\left(\boldsymbol{u}_{S 2}\right) \\
M^{2}\left(\boldsymbol{u}_{S 2}\right) \\
M^{3}\left(\boldsymbol{u}_{S 2}\right)
\end{array}\right)=\mathrm{K}_{b}\left(\boldsymbol{U}_{2}^{B}-\boldsymbol{U}_{2}^{A}\right)-\overline{\mathrm{K}}_{b}\left(\boldsymbol{U}_{1}^{B}-\boldsymbol{U}_{1}^{A}\right)+\left(\begin{array}{c}
Q \\
0 \\
0 \\
0 \\
0 \\
0
\end{array}\right) .
$$

Remark 3. Inner efforts at the end of the first step, the prestressed state, can be evaluated by substituting $\boldsymbol{u}_{S 2}$ with $\boldsymbol{u}_{S 1}$ in (26) and thus read

$$
\left(\begin{array}{l}
F^{1}\left(\boldsymbol{u}_{S 1}\right) \\
F^{2}\left(\boldsymbol{u}_{S 1}\right) \\
F^{3}\left(\boldsymbol{u}_{S 1}\right) \\
M^{1}\left(\boldsymbol{u}_{S 1}\right) \\
M^{2}\left(\boldsymbol{u}_{S 1}\right) \\
M^{3}\left(\boldsymbol{u}_{S 1}\right)
\end{array}\right)=\widetilde{\mathrm{K}}_{b}\left(\boldsymbol{U}_{1}^{B}-\boldsymbol{U}_{1}^{A}\right)+\left(\begin{array}{c}
Q \\
0 \\
0 \\
0 \\
0 \\
0
\end{array}\right)
$$

\subsubsection{Elasticity of the root}

Connections should be considered with elasticity condition while designing assemblies in most cases. This is achieved by controlling the Von Mises yield criterion as stated in [15]

$$
\sqrt{\left(\sigma_{t}+\sigma_{f}\right)^{2}+3 \tau^{2}} \leq 0.9 R e
$$

where $\sigma_{t}$ and $\sigma_{f}$ are respectively the tensile and flexural components of the normal stress, $\tau$ is the shear stress from transversal and torsional forces and $R e$ is the yield stress of the root. The Von Mises criterion is evaluated from inner efforts using theory of elasticity for long beam [19]. It is evaluated at points where it may reach its maximal value: the rim (resp. the center) of the root, where the maximal flexural and torsional components of stress (resp. maximal transversal component stress) lie. Note that the effective implantation length $\ell$ has to be considered to accurately evaluate worst lever-arm effect.

For the sake of simplicity, the yield criterion is squared.

- At the rim of the root $\left(r=r_{b}\right)$

$$
\left(\frac{\sqrt{\left(F^{1}\right)^{2}}}{\pi r_{b}^{2}}+\frac{\sqrt{\left(M^{2}-\ell F^{3}\right)^{2}+\left(M^{3}+\ell F^{2}\right)^{2}}}{\frac{\pi}{4} r_{b}^{3}}\right)^{2}+3\left(\frac{M^{1}}{\frac{\pi}{2} r_{b}^{3}}\right)^{2} \leq(0.9 R e)^{2} .
$$


- At the center of the root $(r=0)$

$$
\left(\frac{F^{1}}{\pi r_{b}^{2}}\right)^{2}+3\left(\frac{4}{3} \frac{\sqrt{\left(F^{2}\right)^{2}+\left(F^{3}\right)^{2}}}{\pi r_{b}^{2}}\right)^{2} \leq(0.9 R e)^{2} .
$$

Remark 4. Given the Euler-Bernoulli condition for long beam, it is very likely that the Von Mises criterion at the center is smaller than the Von Mises criterion at the rim of the root during the optimization process.

Remark 5. Criteria (29) and (30) should be verified for each state of the two-steps process, which requires further research works on the implementation of optimization constraints.

\subsubsection{Fatigue of the root}

Connections should be considered with high cycle fatigue condition while designing assemblies too. For an infinite lifetime in service, long bolts exhibit a maximal allowed conservative stress amplitude given by [15]

$$
\sigma_{a}=0.855\left(\frac{150}{d}+45\right)
$$

Remark 6. Numerical values in (31) are given for a system of units in MPa and mm.

Root fatigue failure appears at engaged threads level, thus, the effective length $\ell$ has to be considered again to accurately evaluate worst lever-arm effect. The fatigue constraint is then given by

$$
\left(\frac{\sqrt{\left(\Delta F^{1}\right)^{2}}}{\pi r_{b}^{2}}+\frac{\sqrt{\left(\Delta M^{2}-\ell \Delta F^{3}\right)^{2}+\left(\Delta M^{3}+\ell \Delta F^{2}\right)^{2}}}{\frac{\pi}{4} r_{b}^{3}}\right)^{2} \leq\left(2 \sigma_{a}\right)^{2}
$$

where $\Delta$ indicates the operating cycle between the two equilibrium states Step 1 and Step 2. For instance $\Delta F^{1}=F^{1}\left(\boldsymbol{u}_{S 2}\right)-F^{1}\left(\boldsymbol{u}_{S 1}\right)$ and likewise for the other inner efforts.

In the sequel, the term "bolt" will always refer to idealized bolt by abuse of language.

\section{Optimization methods}

\subsection{Shape optimization}

\subsubsection{Level-set method}

In this paper, structures are represented with the level-set method as introduced by [28] (see also the textbooks $[27,34]$ ). It offers a large flexibility in topological changes and the boundaries of the structure are explicitly defined. Consider a working domain $\mathcal{D} \subset \mathbb{R}^{3}$ that contains all admissible shapes. A shape (or structure) is parameterized by a function $\psi$ defined on $\mathcal{D}$ such that

$$
\left\{\begin{array}{l}
\psi(\boldsymbol{x})=0 \Longleftrightarrow \boldsymbol{x} \in \partial \Omega \cap \mathcal{D} \\
\psi(\boldsymbol{x})<0 \Longleftrightarrow \boldsymbol{x} \in \Omega \\
\psi(\boldsymbol{x})>0 \Longleftrightarrow \boldsymbol{x} \in(\mathcal{D} \backslash \Omega)
\end{array} .\right.
$$

Thus the boundaries of the structure are defined as the zeros of the level-set function. The boundary of the structure evolves in time according to the transport Hamilton-Jacobi equation

$$
\left\{\begin{aligned}
\frac{\partial \psi}{\partial t}+V|\nabla \psi| & =0 \text { on }[0, \infty[\times \mathcal{D} \\
\psi(t=0, \boldsymbol{x}) & =\psi_{0}(\boldsymbol{x})
\end{aligned}\right.
$$

where $V(t, \boldsymbol{x})$, with $t \in \mathbb{R}^{+}$, is the velocity field. Finally, to avoid undefined tensor when the elasticity problem is solved, the void is replaced by an ersatz material with Hooke's law $\overline{\mathrm{C}}=10^{-3} \mathrm{C}$. 


\subsubsection{Hadamard's boundary variation method}

We use Hadamard's boundary variation method to compute the velocity field $V$ of (34). Introduced by [16], this method describes shape variations. It relies on the notion of differentiation with respect to the position of a shape $\Omega[2,29,37]$. Let $\Omega \subset \mathbb{R}^{3}$ be a given reference shape. Assume that $\Omega$ is an open, smooth and bounded domain of $\mathbb{R}^{3}$. Let $\boldsymbol{\theta} \in W^{1, \infty}\left(\mathbb{R}^{3}, \mathbb{R}^{3}\right)$ be a displacement field of small amplitude, where $W^{1, \infty}\left(\mathbb{R}^{3}, \mathbb{R}^{3}\right)$ is the space of Lipschitz vector fields [2, 37]. The purpose of Hadamard's boundary variation method is to transport the reference domain $\Omega$ into an admissible shape $\Omega_{\theta}=(\boldsymbol{I} \boldsymbol{d}+\boldsymbol{\theta})(\Omega)$. The vector $\boldsymbol{\theta}$ moves slightly all points of $\Omega$ from a location $\boldsymbol{x}$ to a deformed location $\boldsymbol{x}+\boldsymbol{\theta}(\boldsymbol{x})$ as displayed in Fig. 5.

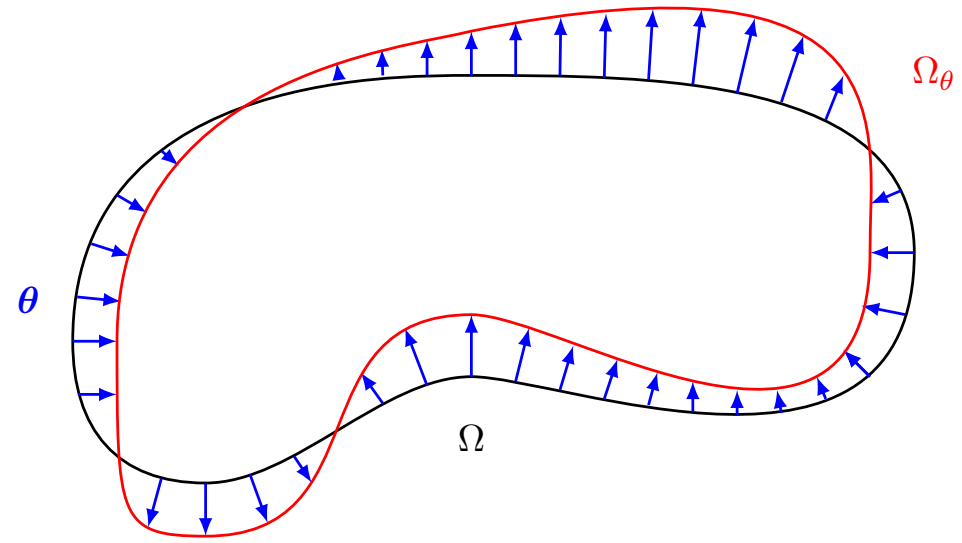

Figure 5: Transport of a domain $\Omega$ to a domain $\Omega_{\theta}$ with the Hadamard's boundary variation method

Definition 1. Let $J(\Omega)$ be a function from the set of admissible shapes $\mathcal{U}_{a d}$ to $\mathbb{R}$. The shape function $J$ is said to be shape differentiable if there exists a continuous linear form $J^{\prime}(\Omega)$ acting on $W^{1, \infty}\left(\mathbb{R}^{3}, \mathbb{R}^{3}\right)$ such that

$$
J((\boldsymbol{I d}+\boldsymbol{\theta})(\Omega))=J(\Omega)+J^{\prime}(\Omega)(\boldsymbol{\theta})+o(\boldsymbol{\theta}), \text { where } \lim _{\boldsymbol{\theta} \rightarrow \mathbf{0}} \frac{|o(\boldsymbol{\theta})|}{\|\boldsymbol{\theta}\|_{W^{1, \infty}\left(\mathbb{R}^{3}, \mathbb{R}^{3}\right)}}=0 .
$$

The function $J^{\prime}(\Omega)$ is called the shape derivative of the shape functional $J$ at $\Omega$. The next proposition gives the example of shape derivative of the compliance, which is useful for the sequel.

Proposition 1. Let $\Omega$ be a smooth, bounded, open set of $\mathbb{R}^{3}$. Let $J$ be the shape functional, called the compliance, from $\mathcal{U}_{a d}$ to $\mathbb{R}$ defined by

$$
J(\Omega)=\int_{\Omega} \mathrm{C} \varepsilon(\boldsymbol{u}): \varepsilon(\boldsymbol{u}) d V,
$$

where $\boldsymbol{u}$ solves (8). Then, $J$ is shape differentiable and it holds

$$
J^{\prime}(\Omega)(\boldsymbol{\theta})=-\int_{\Gamma} \mathrm{C} \varepsilon(\boldsymbol{u}): \varepsilon(\boldsymbol{u}) \boldsymbol{\theta} \cdot \boldsymbol{n} d S, \forall \boldsymbol{\theta} \in W^{1, \infty}\left(\mathbb{R}^{3}, \mathbb{R}^{3}\right),
$$

where $\boldsymbol{n}$ is the outward normal to $\Omega$.

\subsection{Bolt location optimization}

In this paper, the bolt has fixed shape, length and orientation. Bolt location is parameterized by the center $\boldsymbol{x}_{0}$ of its head $\omega_{A}$. It is then optimized with a parametric gradient-based algorithm

$$
\boldsymbol{x}_{0}^{k+1}=\boldsymbol{x}_{0}^{k}-\delta^{k} \frac{\partial J}{\partial \boldsymbol{x}_{0}}\left(\Omega, \boldsymbol{x}_{0}^{k}\right),
$$


where $\boldsymbol{x}_{0}^{k}$ is the center of the sphere $\omega_{A}$ at the iteration $k, \delta^{k}$ is the descent step and $\frac{\partial J}{\partial \boldsymbol{x}_{0}}\left(\Omega, \boldsymbol{x}_{0}^{k}\right)$ is the partial derivative of the objective function with respect to the location $\boldsymbol{x}_{0}$, derived according to Hadamard's boundary variation method. The spheres representing the head and the threads are translated. Therefore, the deformation vector $\boldsymbol{\theta}$ is constant on the given sphere $\omega_{A}$. The following result gives the form of the derivative of the bolt constitutive behavior law and specific mechanical constraints.

Proposition 2. Let $\omega_{A}$ be a smooth bounded, open set of $\mathbb{R}^{3}$. Let $f \in W^{1,1}\left(\mathbb{R}^{3}, \mathbb{R}^{3}\right)$ and $J$ a shape functional from $\mathcal{W}$ to $\mathbb{R}$ defined by

$$
J\left(\Omega, \boldsymbol{x}_{0}\right)=f_{\omega_{A}} f d V=\frac{1}{\left|\omega_{A}\right|} \int_{\omega_{A}} f d V .
$$

Then, $J$ is shape differentiable and it holds

$$
\left\langle\frac{\partial J}{\partial \boldsymbol{x}_{0}}\left(\Omega, \boldsymbol{x}_{0}\right), \boldsymbol{\theta}\right\rangle=J^{\prime}\left(\Omega, \boldsymbol{x}_{0}\right)(\boldsymbol{\theta})=\frac{\boldsymbol{\theta}}{|\omega|} \cdot \int_{\partial \omega_{A}} f \boldsymbol{n} d S, \forall \boldsymbol{\theta} \in W^{1, \infty}\left(\mathbb{R}^{3}, \mathbb{R}^{3}\right),
$$

where $\boldsymbol{n}$ is the outward normal to $\omega_{A}$.

\subsection{Bolt number optimization}

The topological derivative is a notion introduced in $[8,25,12,38,39]$ to indicate where it might be mostly beneficial (for a given objective function) to introduce a small inclusion or a small hole with regards to given boundary conditions. It results from an asymptotic analysis of an objective function with respect to that small inclusion or hole. This notion is extended to a small bolt in [30] in order to determine the advantageous location of an additional bolt in an assembled system.

Briefly stated, the spheres are rescaled by an adimensional factor $\rho>0$ that goes to zero in order to get small perturbations. The topological derivative takes only into account the leading term of $\mathrm{K}_{b}$, the rigidity matrix (1) of the bolt root, with regards to the size of the spheres. This leading rigidity, denoted $\kappa(\rho)$, is the stiffness along the axis of the root, that is to say its traction-compression behavior. Then, the rigidity of the small bolt root and the associated pre-tension amplitude $\Phi(\rho)$ read

$$
\kappa(\rho)=K \rho^{2} \text { and } \Phi(\rho)=Q \rho^{2},
$$

where $K>0$ and $Q>0$. The topological derivative is computed with the displacement fields and the adjoint state of the background domain (i.e. the domain without the supplementary small bolt).

Consider some objective function $J$ to be minimized and denote by $J_{\rho}(\Omega)$ its value in the domain $\Omega$, perturbed by a small bolt (thus, $J_{0}(\Omega)$ is its value in the background domain $\Omega$, without the small bolt). We recall that the center of the head of the bolt is denoted by $\boldsymbol{x}_{0}$.

Definition 2. The objective function $J_{\rho}$ is said to admit a topological derivative $D J\left(\boldsymbol{x}_{0}, \boldsymbol{e}\right)$ at the point $\boldsymbol{x}_{0}$ for a small bolt of direction $\boldsymbol{e}$ and for a pair of inclusions of shape $\omega$, if the following asymptotic expansion holds for small $\rho>0$

$$
J_{\rho}(\Omega)=J_{0}(\Omega)+s(\rho) D J\left(\boldsymbol{x}_{0}, \boldsymbol{e}\right)+o(s(\rho)),
$$

where $s(\rho)$ is a positive scalar function of $\rho$ which satisfies $\lim _{\rho \rightarrow 0} s(\rho)=0$.

If the quantity $D J\left(\boldsymbol{x}_{0}, \boldsymbol{e}\right)$ is negative, it is then favorable to create a small bolt at the point $\boldsymbol{x}_{0}$ in the direction $\boldsymbol{e}$. Its expression is given by the following Theorem (cf. [32]) 
Theorem 1. Let $\omega$ be the unit ball of $\mathbb{R}^{3}$. Let us set

$$
\begin{aligned}
& \boldsymbol{U}_{S 1}=\boldsymbol{u}_{S 1}\left(\boldsymbol{x}_{0}+\ell \boldsymbol{e}\right)-\boldsymbol{u}_{S 1}\left(\boldsymbol{x}_{0}\right), \\
& \boldsymbol{U}_{S 2}=\boldsymbol{u}_{S 2}\left(\boldsymbol{x}_{0}+\ell \boldsymbol{e}\right)-\boldsymbol{u}_{S 2}\left(\boldsymbol{x}_{0}\right), \\
& \boldsymbol{P}_{S 2}=\boldsymbol{p}_{S 2}\left(\boldsymbol{x}_{0}+\ell \boldsymbol{e}\right)-\boldsymbol{p}_{S 2}\left(\boldsymbol{x}_{0}\right),
\end{aligned}
$$

where $\boldsymbol{u}_{S 1}, \boldsymbol{u}_{S 2}$ and $\boldsymbol{p}_{S 2}$ are respectively the displacement fields of the background Step 1, Step 2 and the adjoint state of the background adjoint problem of Step 2. The objective function $J_{\rho}$ admits a topological asymptotic expansion of the form (40), that reads

$$
J_{\rho}(\Omega)=J_{0}(\Omega)+\rho^{2}\left(K\left(\boldsymbol{U}_{S 2}-\boldsymbol{U}_{S 1}\right) \cdot \boldsymbol{e}+Q\right) \boldsymbol{P}_{S 2} \cdot \boldsymbol{e}+O\left(\rho^{3}\right) .
$$

\section{Numerical illustrations}

\subsection{Academic use case}

\subsubsection{Setting of the problem}

This academic $3 \mathrm{~d}$ use case is a precursor to the more significant and complete simplified industrial use case developed in Section 4.2. Let $\Omega$ be a cube of side $L=100 \mathrm{~mm}$ with a vertical crack on the middle, as pictured in Fig. 6. The cube is clamped on the left side and a sinusoidal horizontal force $\boldsymbol{g}=\left(\sin \left(\frac{\pi z}{2}\right) \times 10^{5}, 0,0\right)$ is applied on a thin surface of $2 \mathrm{~mm}$ height of the right side. Young modulus and Poisson's ratio are $E_{\text {cube }}=210 \mathrm{GPa}$ and $\nu_{\text {cube }}=0.3$. The sliding contact condition of Section 2.3 is applied on the crack. The mesh initially contains 31569 tetrahedral elements. In this use case, the properties of the bolt are :

- radius $r_{b}=5 \mathrm{~mm}$

- length $\ell=50 \mathrm{~mm}$

- Young modulus $E=172.5 \mathrm{GPa}$

- Poisson's ratio $\nu=0.3$

- Pre-tension force $Q=25000 \mathrm{~N}$

A surrounding of material is added around each sphere. This non-design domain for the structure is spherical-shaped and twice the size of the sphere. It ensures the diffusion of efforts into the system.

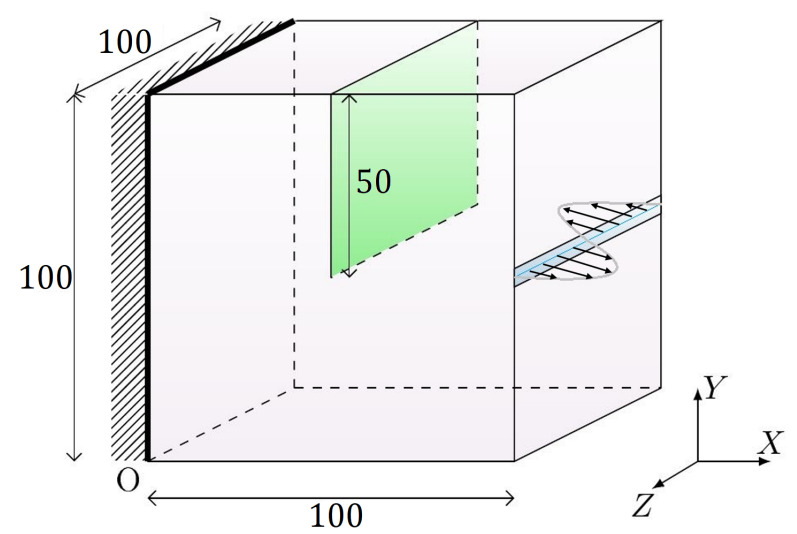

Figure 6: Setting of a cube with a crack

The problem is initialized without bolts. We use the topological derivative to place the first bolt. Then we perform a structure optimization with this fixed bolt as a reference. After that, a coupled optimization of both structure and bolt location is performed with two alternating strategies. 


\subsubsection{Test of the topological derivative}

Given the cube size $(L=100 \mathrm{~mm})$ and the spheres radius $\left(r_{b}=5 \mathrm{~mm}\right)$, the bolt is small enough to justify the use of topological derivative. The topological derivative is used to place one bolt oriented along $\boldsymbol{X}$ axis. The goal is to decrease the compliance $J(\Omega)=\int_{\Gamma_{N}} \boldsymbol{g} \cdot \boldsymbol{u} d S$. The topological derivative indicates the best location to put a 1 DOF bolt. We put directly a 6 DOFs bolt at the location given by the topological derivative, that is $\boldsymbol{x}_{0}=(-26.8,32.5,32.4)$ (see Fig. 7). The initial and the final compliance, resp. without and with the bolt, are $J_{0}(\Omega)=102 \mathrm{~J}$ and $J_{\rho}(\Omega)=95 \mathrm{~J}$. Then the location given by the topological derivative, reminding it considers only the main DOF of the bolt, provides improvements even when a 6 DOFs bolt is used in the elasticity problem.

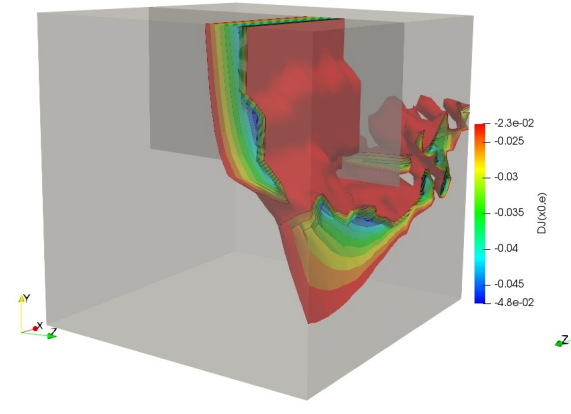

(a)

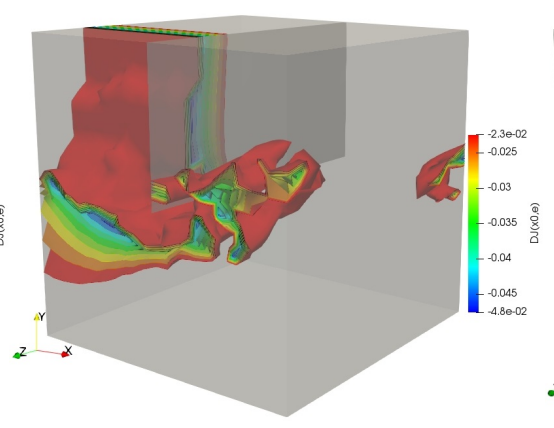

(b)

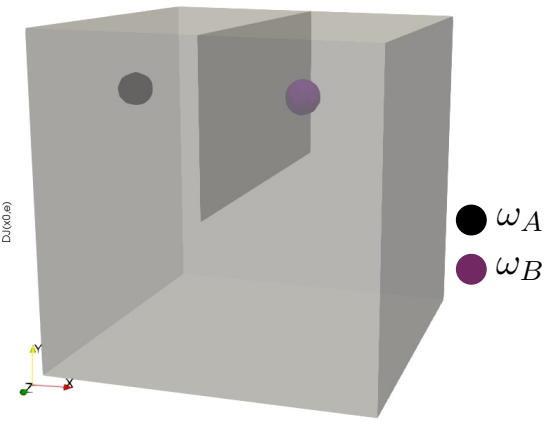

(c)

Figure 7: Iso-values of the topological derivative (a), (b) and the optimal location of the bolt (c)

\subsubsection{Structure optimization with a fixed bolt}

Let us optimize the structure where the bolt remains fixed and a technological constraint is imposed to the bolt. The structure is initialized with holes and the bolt is at the location given by the topological derivative (see Fig. 8). The problem is to minimize the volume $V(\Omega)$ under a constraint
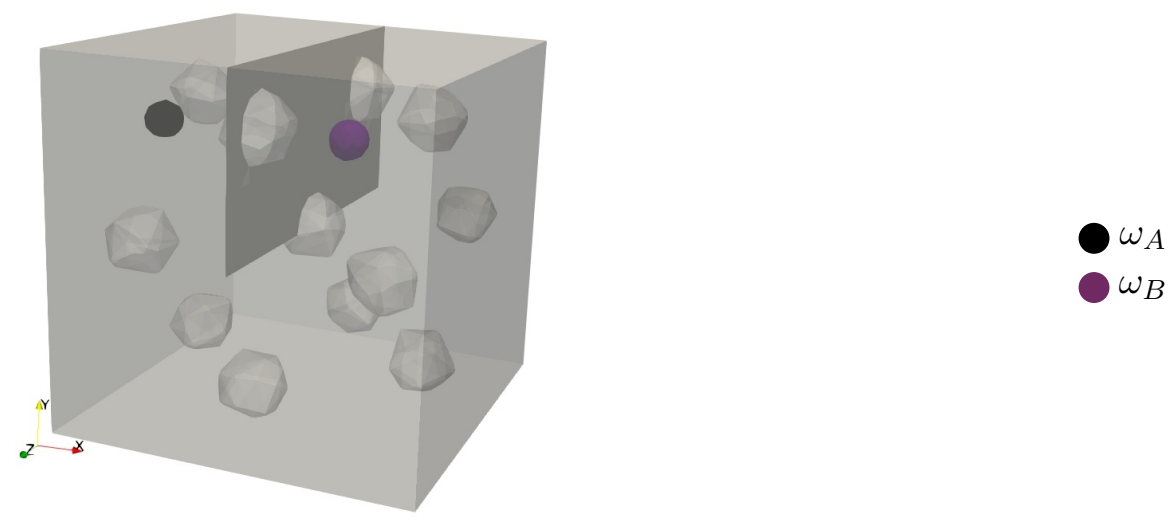

Figure 8: Initial topology and bolt location

on the compliance $C(\Omega)$ and on the Von Mises at the rim of the bolt $\operatorname{VMR}(\Omega)$. It reads

$$
\begin{gathered}
\min _{\Omega \in \mathcal{U}_{a d}} V(\Omega), \\
C(\Omega) \leq C_{0} \\
\operatorname{VMR}(\Omega) \leq \operatorname{VM}_{0}
\end{gathered}
$$

where the constraint bounds are $C_{0}=110 \mathrm{~J}$ and $\mathrm{VM}_{0}=656 \times 10^{5} \mathrm{~Pa}^{2}$. Optimization constraints are taken into account with an Augmented Lagrangian algorithm. Therefore, the problem (43) is 
rewritten as

$$
\begin{aligned}
\min _{\Omega \in \mathcal{U}_{a d}} \max _{\boldsymbol{\alpha} \geq \mathbf{0}}\{\mathcal{L}(\Omega)=V(\Omega) & +\alpha_{C}\left(C(\Omega)-C_{0}\right)+\frac{\beta_{C}}{2}\left(C(\Omega)-C_{0}\right)^{2} \\
& \left.+\alpha_{\mathrm{VMR}}\left(\operatorname{VMR}(\Omega)-\mathrm{VM}_{0}\right)+\frac{\beta_{\mathrm{VMR}}}{2}\left(\operatorname{VMR}(\Omega)-\mathrm{VM}_{0}\right)^{2}\right\},
\end{aligned}
$$

where $\boldsymbol{\alpha}=\left(\alpha_{C}, \alpha_{\mathrm{VMR}}\right)$.

The optimal shape is given in Fig. 9. The final volume is $V(\Omega)=5.40 \times 10^{5} \mathrm{~mm}^{3}$. The Von Mises constraint is saturated while the compliance $C(\Omega)=104 \mathrm{~J}$ is below the threshold.

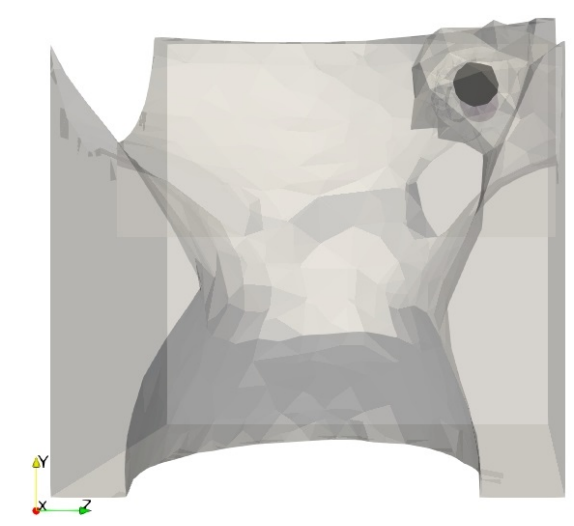

(a)

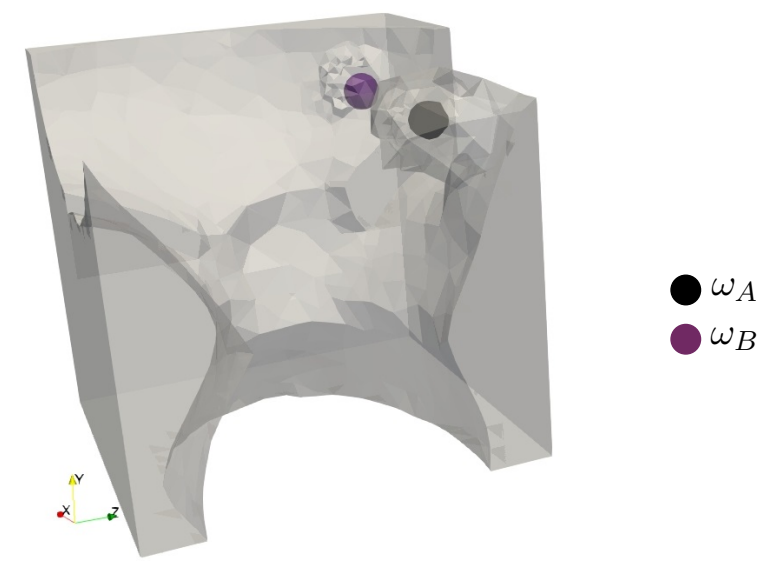

(b)

Figure 9: Optimal structure for the optimization problem (43): volume minimization, with compliance constraint and Von Mises constraint at the rim of the bolt root

\subsubsection{Coupled optimization of structure and bolt location}

Consider the initialization of Fig. 8. We perform now a coupled optimization of both the structure and the bolt location to solve the problem (44) with 3 iterations of structure advection for 1 iteration of parametric gradient for the location, until convergence.

Remark 7. The bolt is movable in the following optimization problem. Since the initial mesh is coarse enough to save computation time, we need to remesh around the spheres for each location change to ensure a correct computation of the displacement field on the bolt extremities. Consequently, numerical results may present some fluctuations. The mesh is refined around the spheres with the open source library mmg3d [10].

The optimal structure and bolt location are shown in Fig. 10. The final volume is $V(\Omega)=$ $4.40 \times 10^{5} \mathrm{~mm}^{3}$. This is quite an improvement compared to the structure optimized with a fixed bolt for which the final volume was $V(\Omega)=5.40 \times 10^{5} \mathrm{~mm}^{3}$. The optimal bolt location is $\boldsymbol{x}_{0}=(-10.6,32.7,33.1)$, which corresponds to an amplitude of displacement of $16.2 \mathrm{~mm}$.

The convergence history is given in Fig. 11. We deliberately display all iterations, even those which have been rejected. It is quite noticeable how the brutal violation of the Von Mises constraint leads to an important response of the bolt location, and not of the structure around iteration 100. The parametric optimization of the bolt location reduces the constraints violation and proves its efficiency for the integration of specific mechanical constraint on the bolt. Actually, the change of location does not affect the oscillations of the compliance constraint around the bound. Note that these oscillations are customary with the Augmented Lagrangian method.

We also solve the same optimization problem with a strategy of 1 iteration of structure advection for 1 iteration of parametric gradient. The optimal structure and bolt location are given in Fig. 12. 


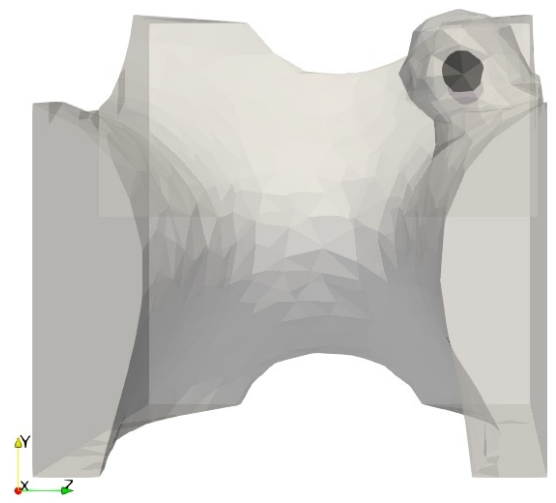

(a)

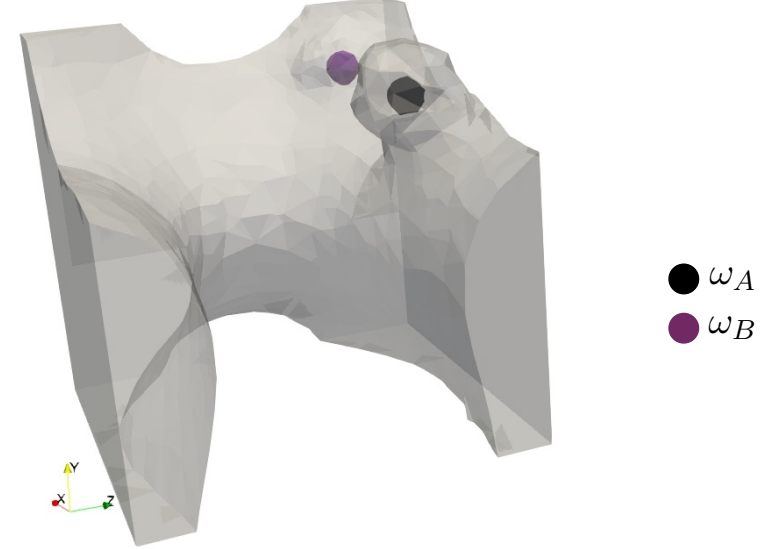

(b)

Figure 10: Optimal structure and bolt location for the problem of volume minimization under a compliance constraint and Von Mises criterion at the rim of the bolt root with an alternating strategy of 3 iterations of structure advection and 1 iteration of parametric gradient for bolt location
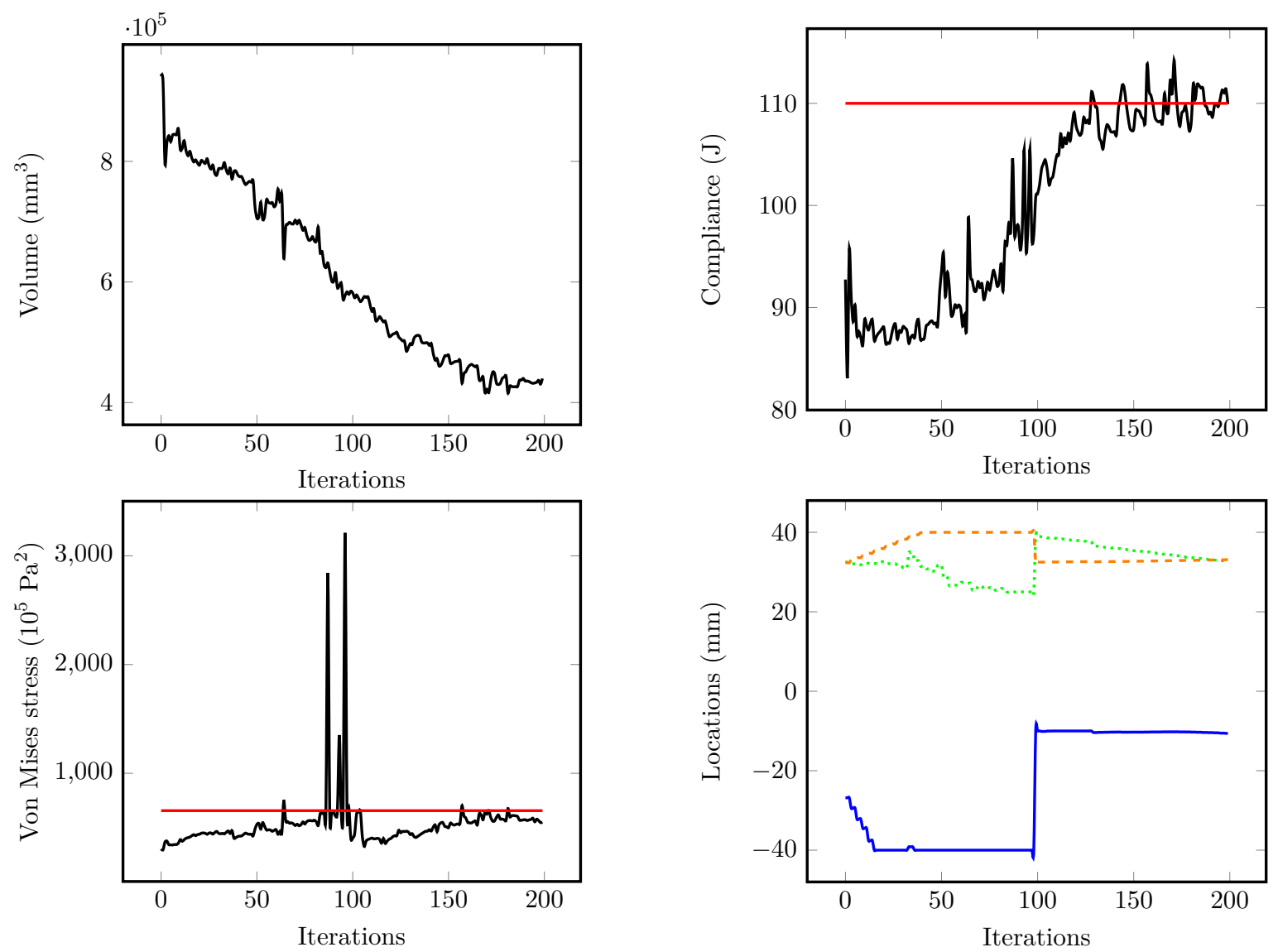

Figure 11: Convergence history of the volume minimization problem under several constraints (compliance and Von Mises at the rim of the bolt root, threshold in red) with an alternating strategy of 3 iterations of structure advection and 1 iteration of parametric gradient for bolt location (X in blue solid line, $\mathrm{Y}$ in green dotted line and $\mathrm{Z}$ in orange dashed line)

The final volume of $V(\Omega)=4.25 \times 10^{5} \mathrm{~mm}^{3}$, which is a better result, of about $3.5 \%$, than the one given by the previous alternating strategy. The optimal bolt is located at $\boldsymbol{x}_{0}=(-10.1,32.0,33.2)$, 
which corresponds to an amplitude of displacement of $16.7 \mathrm{~mm}$ from initial location, and $0.87 \mathrm{~mm}$ away from final location of previous optimization.

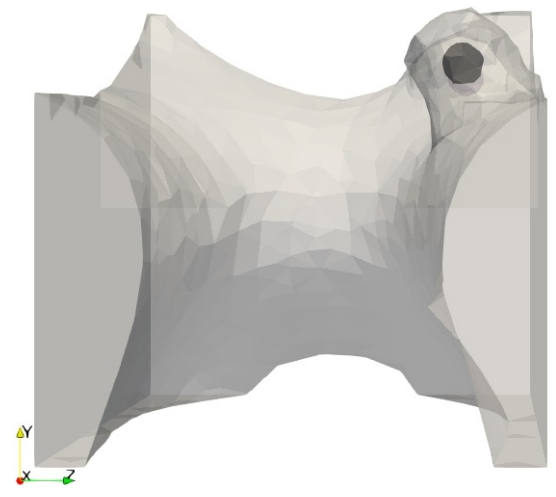

(a)

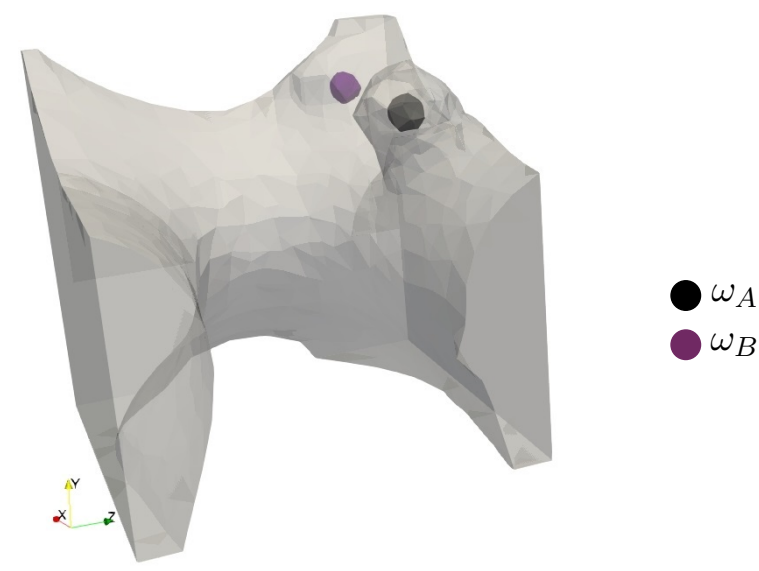

(b)

Figure 12: Optimal structure and bolt location for the problem of volume minimization under a compliance constraint and Von Mises criterion at the rim of the bolt root with an alternating strategy of 1 iteration of structure advection for 1 iteration of parametric gradient for bolt location

The convergence history is shown in Fig. 13. We still display iterations which have been rejected. A brutal decrease of the volume provokes an important increase of the compliance and the Von Mises response. The coupling proves its efficiency to bring each response back into the admissible domain : the structure advection acts for the compliance and the parametric gradient acts mainly for the Von Mises criterion.

Remark 8. The iterations frequency between structure optimization and locations optimization may have a great impact on the optimization process. In [30] (Chapter 5, Section 5.3.2, Remark 48), it has been noticed that final topology of a $2 D$ use case is strongly redesigned, switching from a strategy of 4 iterations of structure for 1 iteration of locations which favors the structure and disregards the connections behavior, to a strategy of 1 structure for 1 location which allows equal efficiency between both optimization ingredients.

\subsection{Simplified industrial use case}

\subsubsection{Setting of the problem}

This section is devoted to a simplified model of an accessories bracket assembly taken from a diesel engine (cf. Fig. 14). This simplified model has the characteristic dimensions of a reference model given by Renault (cf. Fig. 15). Accessories are an alternator, a belt tensioning roller and an air conditioning (AC) compressor. Perfect bonding is assumed between the accessories and the bracket. The accessories bracket insures their positioning and their fastening to the crankcase. The assembly is the union of all accessories plus the bracket plus the crankcase. In the sequel, the accessories and the crankcase are not optimized.

The assembly $\Omega_{A S B}$ is made of disjointed parts

$$
\Omega_{A S B}=\Omega \cup \Omega_{N D S} \cup \Omega_{A C C} \cup \Omega_{C K C},
$$

namely the design-space $\Omega$ and the non design-space $\Omega_{N D S}$ of the bracket (resp. in brown and in red in Fig. 14), the accessories $\Omega_{A C C}$ and the crankcase $\Omega_{C K C}$. Boundaries, depicted in Fig. 16, are defined by

$$
\partial \Omega_{A S B}=\Gamma_{T F} \cup \Gamma_{D} \cup \Gamma_{N},
$$



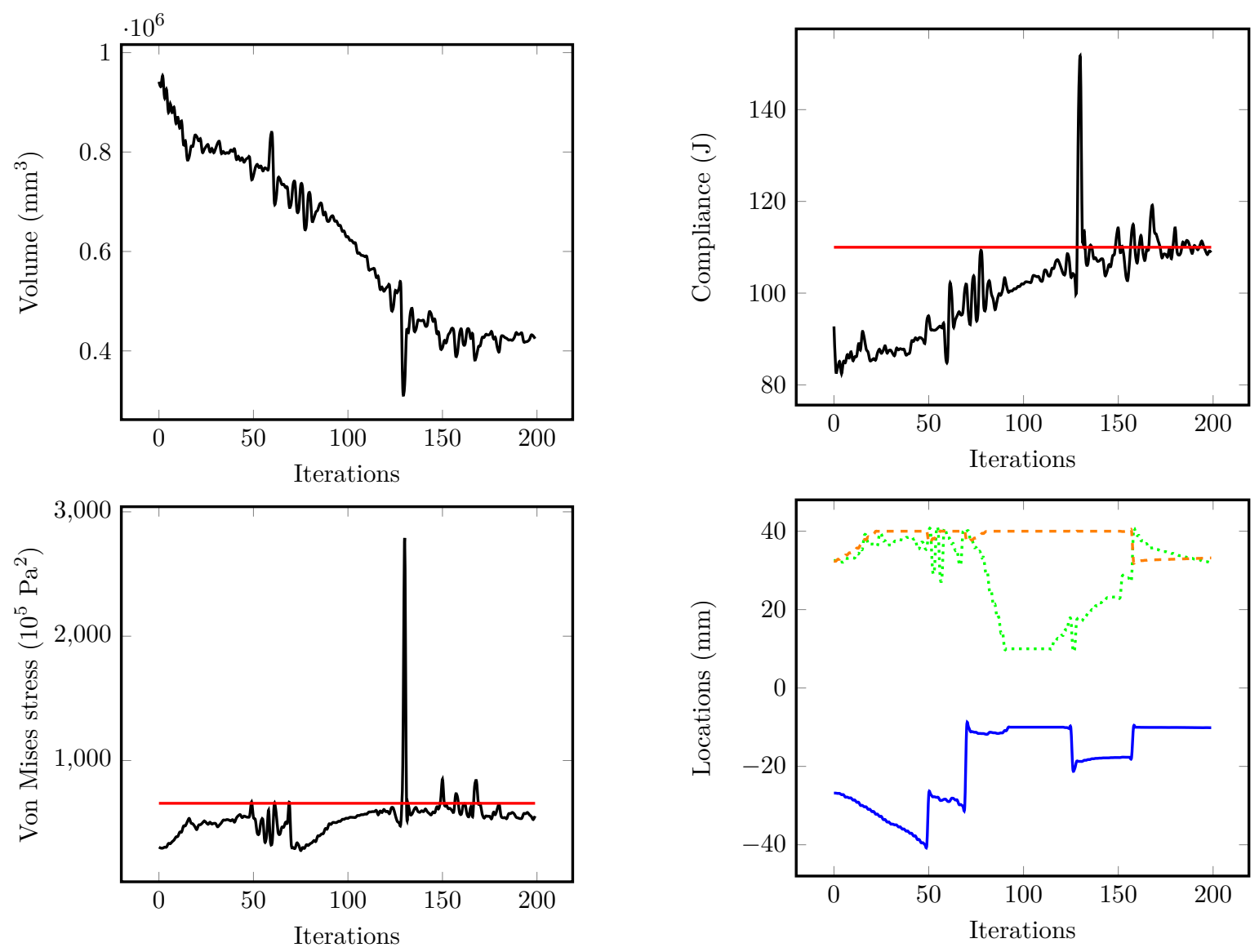

Figure 13: Convergence history of the volume minimization problem under several constraints (compliance and Von Mises at the rim of the bolt root, threshold in red) with an alternating strategy of 1 iteration of structure advection and 1 iteration of parametric gradient for bolt location (X in blue solid line, $\mathrm{Y}$ in green dotted line and $\mathrm{Z}$ in orange dashed line)

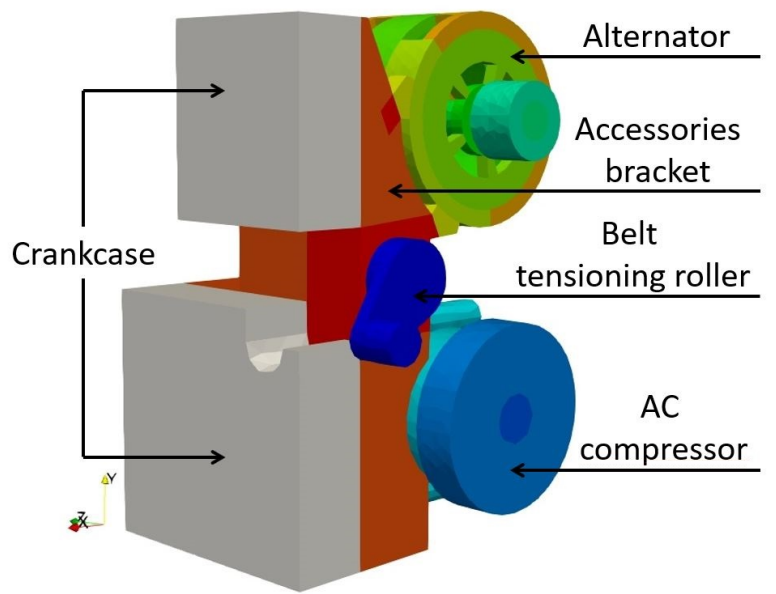

Figure 14: Simplified accessories and bracket

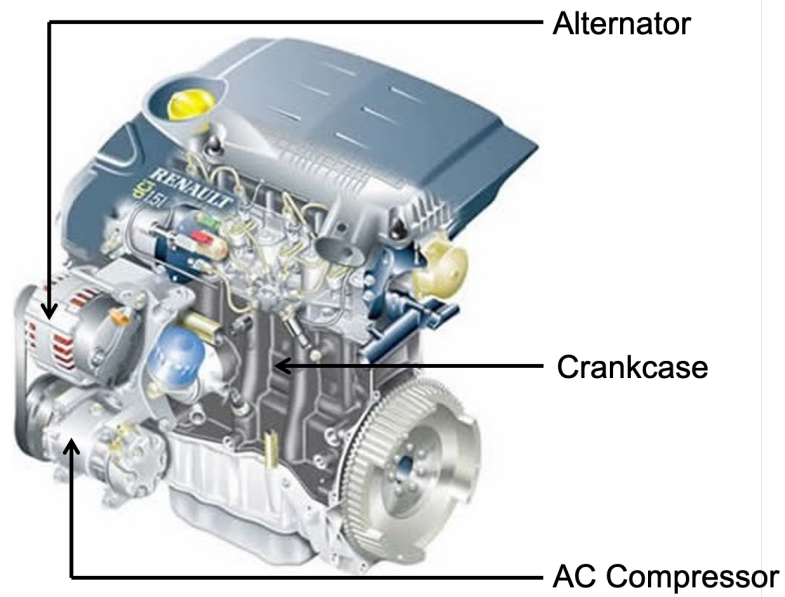

Figure 15: Reference engine

where $\Gamma_{T F}$ is the traction-free boundary and $\Gamma_{D}$ corresponds to clamped zones. Surface loads $\boldsymbol{g}$ on the accessories pulleys are applied simultaneously on 3 different parts of $\Gamma_{N}$. Loads $\boldsymbol{g}$ are then split into $\boldsymbol{g}_{A L T}, \boldsymbol{g}_{B T R}$ and $\boldsymbol{g}_{C O M P}$ as displayed in Fig. 17. They correspond to integrated contact force of the tensioned belt on each pulley. The in-service mechanical analysis problem is then a single and 
static load case. Since the accessories and the crank-case are not optimized, it is better to separate the traction-free boundary of the design-space of the bracket, denoted $\Gamma$, from the traction-free boundaries of accessories, that is $\Gamma_{T F} \backslash \Gamma$. We assume perfect bounding between accessories and the bracket while the bracket is bolted onto the crankcase. The sliding contact condition described in Section 2.3 is applied between the bracket and the crank-case.

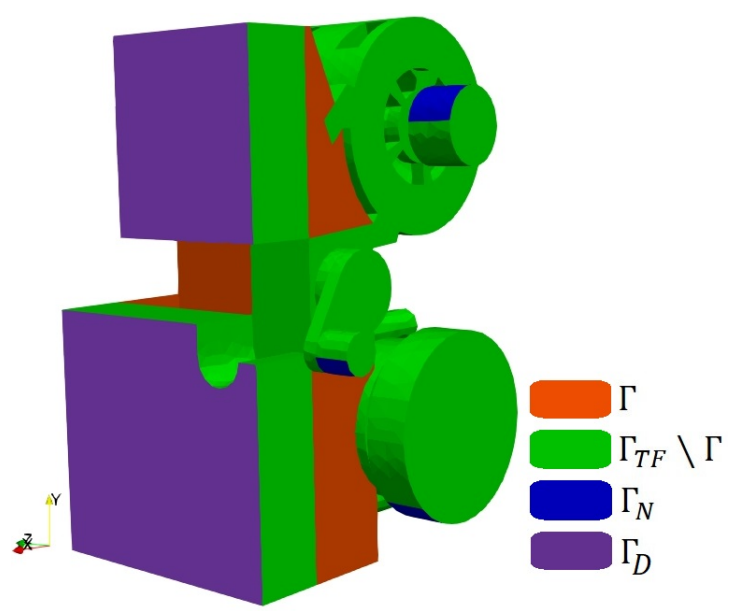

Figure 16: Boundaries of the sub-parts

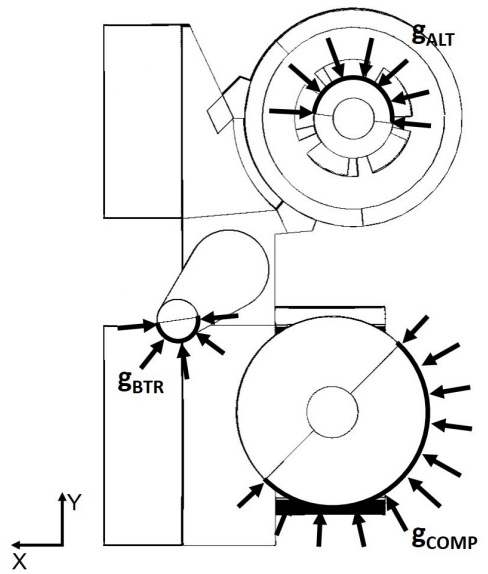

Figure 17: Load case

Bolts orientation is fixed to satisfy industrial manufacturing constraints. All bolts are parallel to the axis $\boldsymbol{X}$, and their head and threads (resp. $\omega_{A}$ and $\omega_{B}$ ) are equally distant from the contact interface plane between the bracket and the crankcase. We consider $n$ identical bolts with the following properties, corresponding to M10 $\times 1.5$ standard bolt :

radius $r_{b}=4.3 \mathrm{~mm}$, length $\ell=50 \mathrm{~mm}$, Young modulus $E=176 \mathrm{GPa}$, Poisson's ratio $\nu=0.3$, Pre-tension force $Q=20000 \mathrm{~N}$ and Yield stress of the root $R e=900 \mathrm{MPa}$.

\subsubsection{Optimization problem}

The problem is to minimize the volume of the bracket $V(\Omega)=\int_{\Omega} d V$ under a constraint on the compliance of the assembly $C(\Omega)=\int_{\Gamma_{N}} \boldsymbol{g} \cdot \boldsymbol{u}_{S 2} d S$. Von Mises at the rim and at the center of the bolt root and fatigue constraints are implemented for each bolt. For the sake of simplicity, we consider for fatigue cycle that belt tension undergoes variations of full amplitude $\boldsymbol{g}$, which is not the case in real in-service conditions.

Remark 9. We use the unit system of ( $m N, k P a, m m)$. We recall that all Von Mises and fatigue constraints, and so their corresponding bounds, are squared. Therefore, they are given in $k P a^{2}$.

Denote $\boldsymbol{X}=\left(\boldsymbol{x}_{1}, \ldots, \boldsymbol{x}_{n}\right)$ where $\boldsymbol{x}_{i}, 1 \leq i \leq n$, is the center of the head of the bolt $i$. The coupled optimization of both the bracket structure and the bolts location reads

$$
\begin{aligned}
& \min _{(\Omega, \boldsymbol{X}) \in \mathcal{U}_{a d}} V(\Omega), \\
& \text { s.t. }\left\{\begin{array}{l}
C(\Omega, \boldsymbol{X}) \leq C_{0} \\
\operatorname{VMR}_{i}^{2}\left(\Omega, \boldsymbol{x}_{i}\right) \leq \mathrm{VM}_{0}^{2}, \forall i \in \llbracket 1, n \rrbracket \\
\operatorname{VMC}_{i}^{2}\left(\Omega, \boldsymbol{x}_{i}\right) \leq \mathrm{VM}_{0}^{2}, \forall i \in \llbracket 1, n \rrbracket \\
F_{i}^{2}\left(\Omega, \boldsymbol{x}_{i}\right) \leq F_{0}^{2}, \forall i \in \llbracket 1, n \rrbracket
\end{array}\right.
\end{aligned}
$$

where constraints bounds are 
- Compliance : $C_{0}=325000 \mu \mathrm{J}$,

- Von Mises : $\mathrm{VM}_{0}^{2}=6.56 \times 10^{11} \mathrm{kPa}^{2}$,

- Fatigue : $F_{0}^{2}=3.5 \times 10^{10} \mathrm{kPa}^{2}$.

Remark 10. The Von Mises bound $\mathrm{VM}_{0}^{2}$ is equal to $(0.9 R e)^{2}$ (see (28)) The fatigue bound $F_{0}^{2}$ is equal to $\left(2 \sigma_{a}\right)^{2}$ (see (32)). However, we recall that fatigue cycle of our simplified model undergoes larger amplitude compared to realistic in-service conditions, the fatigue limit value, $\sigma_{a}$, has to be numerically adapted and thus differs from [15].

The optimization problem (45) is computed with the Augmented Lagrangian method. It is thus rewritten as

$$
\begin{aligned}
& \min _{(\Omega, \boldsymbol{X}) \in \mathcal{U}_{a d}} \max _{\boldsymbol{\alpha} \in \mathbb{R}^{3 n+1}}\left\{\mathcal{L}(\Omega, \boldsymbol{X})=V(\Omega)+\alpha_{C} \max \left(C(\Omega, \boldsymbol{X})-C_{0},-\frac{\alpha_{C}}{\beta_{C}}\right)+\frac{\beta_{C}}{2} \max \left(C(\Omega, \boldsymbol{X})-C_{0},-\frac{\alpha_{C}}{\beta_{C}}\right)^{2}\right. \\
& +\sum_{i=1}^{n} \alpha_{\mathrm{VMR}, i} \max \left(\operatorname{VMR}_{i}^{2}\left(\Omega, \boldsymbol{x}_{i}\right)-\mathrm{VM}_{0}^{2},-\frac{\alpha_{\mathrm{VMR}, i}}{\beta_{\mathrm{VMR}, i}}\right)+\frac{\beta_{\mathrm{VMR}, i}}{2} \max \left(\mathrm{VMR}_{i}^{2}\left(\Omega, \boldsymbol{x}_{i}\right)-\mathrm{VM}_{0}^{2},-\frac{\alpha_{\mathrm{VMR}, i}}{\beta_{\mathrm{VMR}, i}}\right)^{2} \\
& +\sum_{i=1}^{n} \alpha_{\mathrm{VMC}, i} \max \left(\operatorname{VMC}_{i}^{2}\left(\Omega, \boldsymbol{x}_{i}\right)-\mathrm{VM}_{0}^{2},-\frac{\alpha_{\mathrm{VMC}, i}}{\beta_{\mathrm{VMC}, i}}\right)+\frac{\beta_{\mathrm{VMC}, i}}{2} \max \left(\mathrm{VMC}_{i}^{2}\left(\Omega, \boldsymbol{x}_{i}\right)-\mathrm{VM}_{0}^{2},-\frac{\alpha_{\mathrm{VMC}, i}}{\beta_{\mathrm{VMC}, i}}\right)^{2} \\
& \left.+\sum_{i=1}^{n} \alpha_{F, i} \max \left(F_{i}^{2}\left(\Omega, \boldsymbol{x}_{i}\right)-F_{0}^{2},-\frac{\alpha_{F, i}}{\beta_{F, i}}\right)+\frac{\beta_{F, i}}{2} \max \left(F_{i}^{2}\left(\Omega, \boldsymbol{x}_{i}\right)-F_{0}^{2},-\frac{\alpha_{F, i}}{\beta_{F, i}}\right)^{2}\right\}
\end{aligned}
$$

where $\boldsymbol{\alpha}=\left(\alpha_{C}, \alpha_{\mathrm{VMR}, 1}, \ldots, \alpha_{\mathrm{VMR}, n}, \alpha_{\mathrm{VMC}, 1}, \ldots, \alpha_{\mathrm{VMC}, n}, \alpha_{F, 1}, \ldots, \alpha_{F, n}\right)$ are Lagrange multipliers and $\boldsymbol{\beta}=\left(\beta_{C}, \beta_{\mathrm{VMR}, 1}, \ldots, \beta_{\mathrm{VMR}, n}, \beta_{\mathrm{VMC}, 1}, \ldots, \beta_{\mathrm{VMC}, n}, \beta_{F, 1}, \ldots, \beta_{F, n}\right)$ are penalty parameters.

The coupled optimization involves two types of design-variables and thus requires two types of design spaces as displayed in Fig. 18. The design space of the structure is naturally the entire bracket. In the sequel, the structure is always initialized to the completely filled bracket design space. Bolts translate inside the area delimited by the white lines in Fig. 18 without non-overlapping condition. Finally, in the following optimizations, we alternate between one structure iteration and one bolt-location iteration.

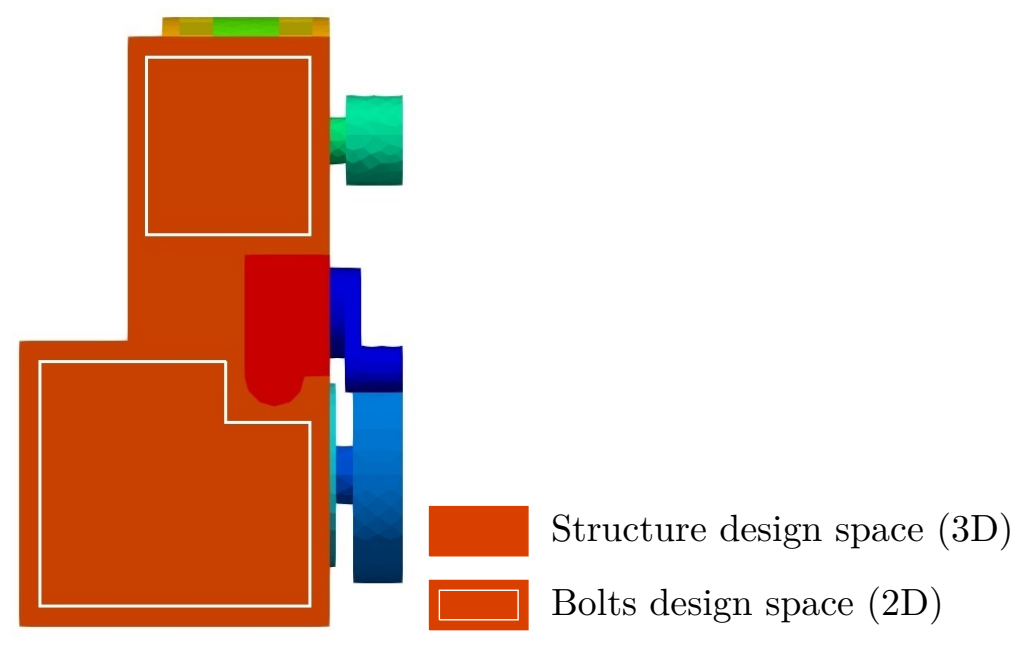

Figure 18: Structure and bolts design spaces 


\subsubsection{Reference optimization: structure alone}

Seven bolts are placed according to a user's proposition which is the reference bolts distribution and number (see Fig. 19). For comparison purposes, we perform a topology optimization to solve the problem (46) where the only design variable is the structure of the bracket while these seven bolts are fixed. The final shape of the bracket is given in Fig. 20. The bracket has a final volume of $V(\Omega)=1.13 \times 10^{6} \mathrm{~mm}^{3}$. All optimization constraints are respected. The compliance bound is not reached. All Von Mises constraints are significantly below the threshold. The only saturated constraint is the fatigue of bolt 4 (see Fig. 19), which leads to the convergence of the structure (see Fig. 24). Moreover, material is uniformly distributed between the upper and the lower part of the bracket. The thickness is almost constant from top to bottom. The bracket structure is smooth and thus appears to be manufacturable.
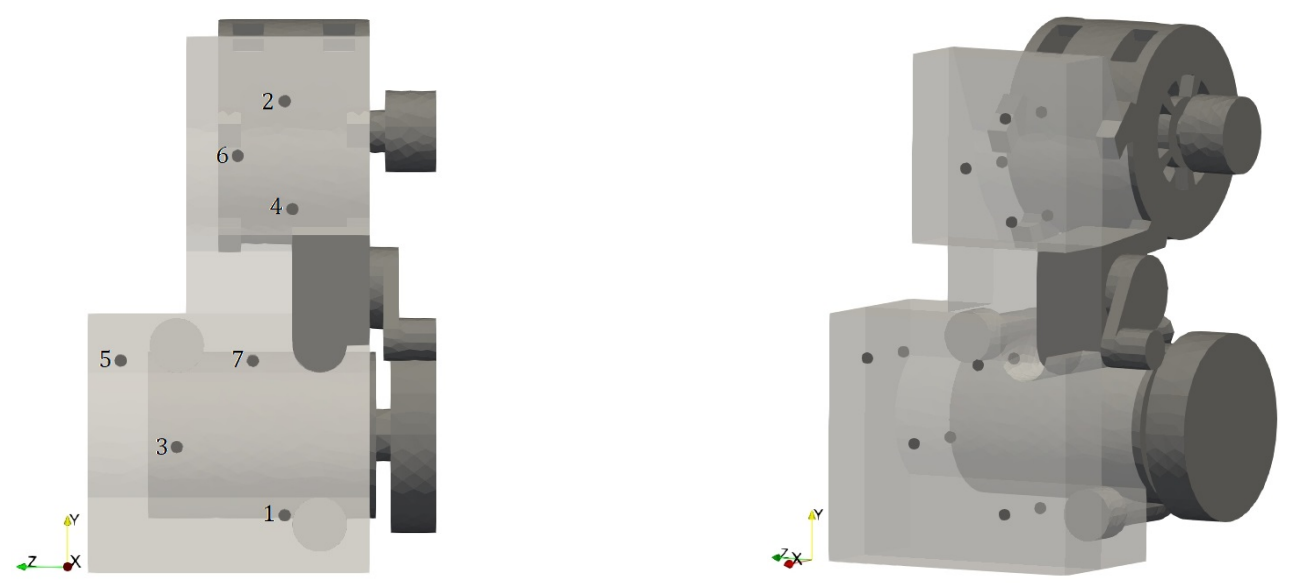

Figure 19: User's proposition for the distribution of 7 bolts (in black)

Remark 11. The optimization problem (46) without any constraints on the 7 bolts has been performed in [30]. It has been then noticed that the final shape exhibits an unbalanced material distribution between upper part (thick) and lower part (thin) of the bracket.

\subsubsection{Coupled optimization}

\section{Use of five bolts}

Let us start with bolts 1, 2, 3 and 4 from the user's proposition (see Fig. 19). The mechanical analysis shows that some bolts mechanical constraints are violated (see [30], Section 9.2.4). Then we decide to firstly apply the topological derivative to decrease the compliance of the assembly. Negative iso-values of the topological derivative are given in Fig. 21. The most relevant area (blue) places the fifth bolt at the upper left hand-side of the bracket (see Fig. 22). The new compliance (with five bolts) is $C(\Omega)=305000 \mu \mathrm{J}$, which is under the threshold and all mechanical constraints on bolts are satisfied too.

Let us now perform a coupled optimization of the structure and bolts location to solve the problem (46). Final shape and bolts location are given in Fig. 23. The compliance bound is reached and all bolts mechanical constraints are satisfied. Bolts location has changed with a minimum displacement amplitude of $0.09 \mathrm{~mm}$ for bolt 4 and a maximum of $5.24 \mathrm{~mm}$ for bolt 3 . The bracket is again smooth with a rather regular material distribution between the upper and the lower parts. Finally, the bracket has a final volume of $V(\Omega)=9.56 \times 10^{5} \mathrm{~mm}^{3}$. It represents a reduction of about $15.5 \%$ compared to the reference result with seven fixed bolts. Convergence history of the volume, the compliance and the fatigue of bolts 1 and 4 are compared in Fig. 24 for the problem with 7 fixed bolts and with 5 optimized bolts. 

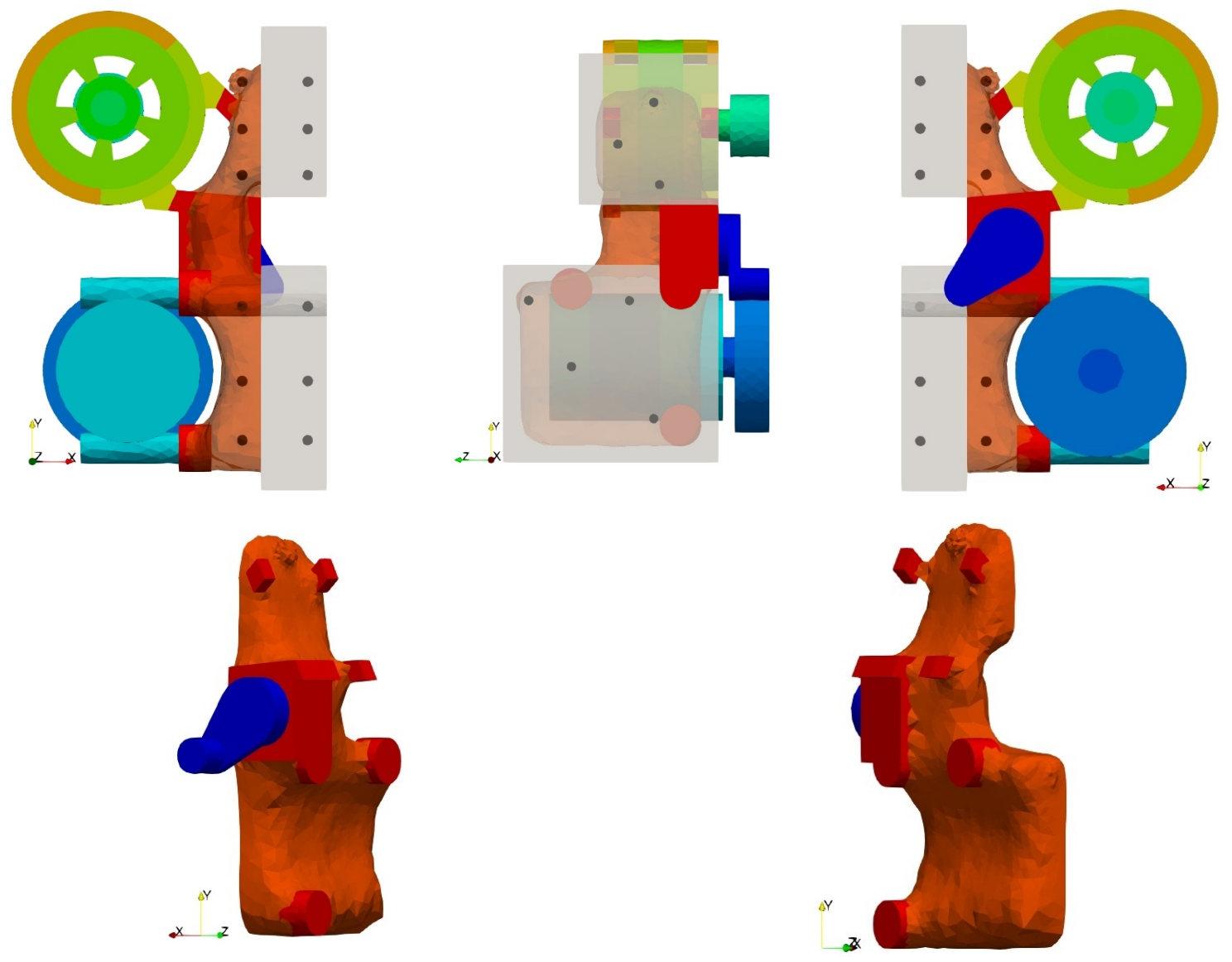

Figure 20: Optimal shape with 7 fixed bolts
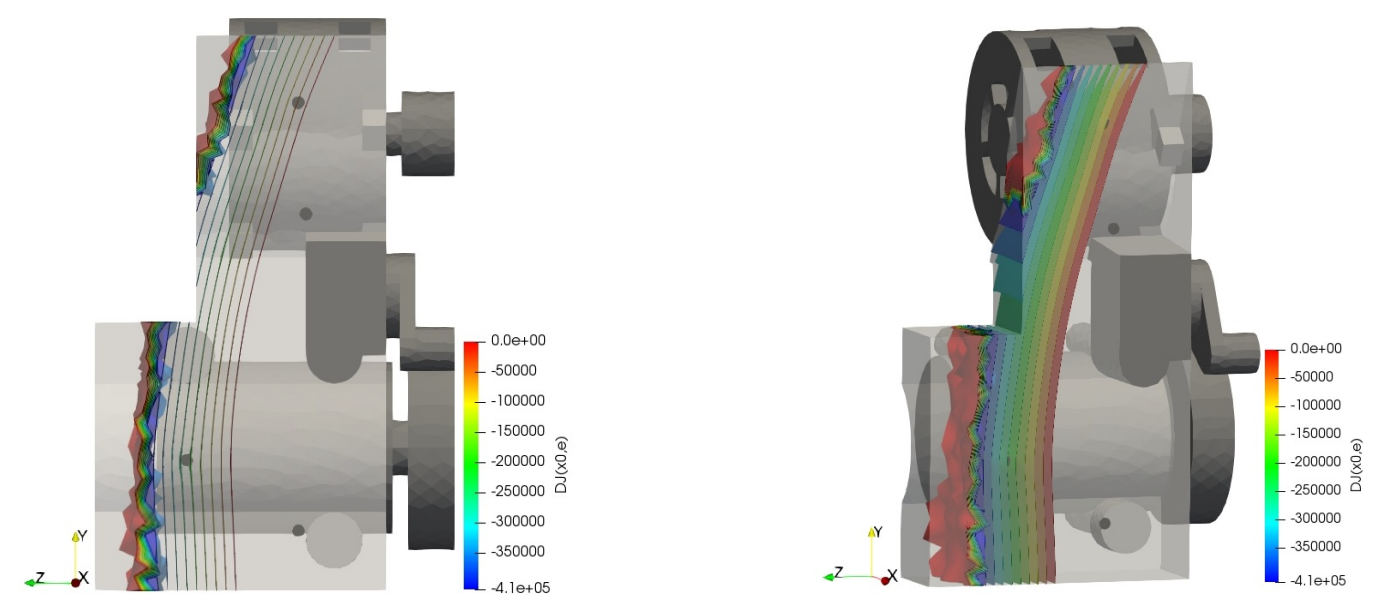

Figure 21: Iso-values of the topological derivative on the assembly with four bolts 

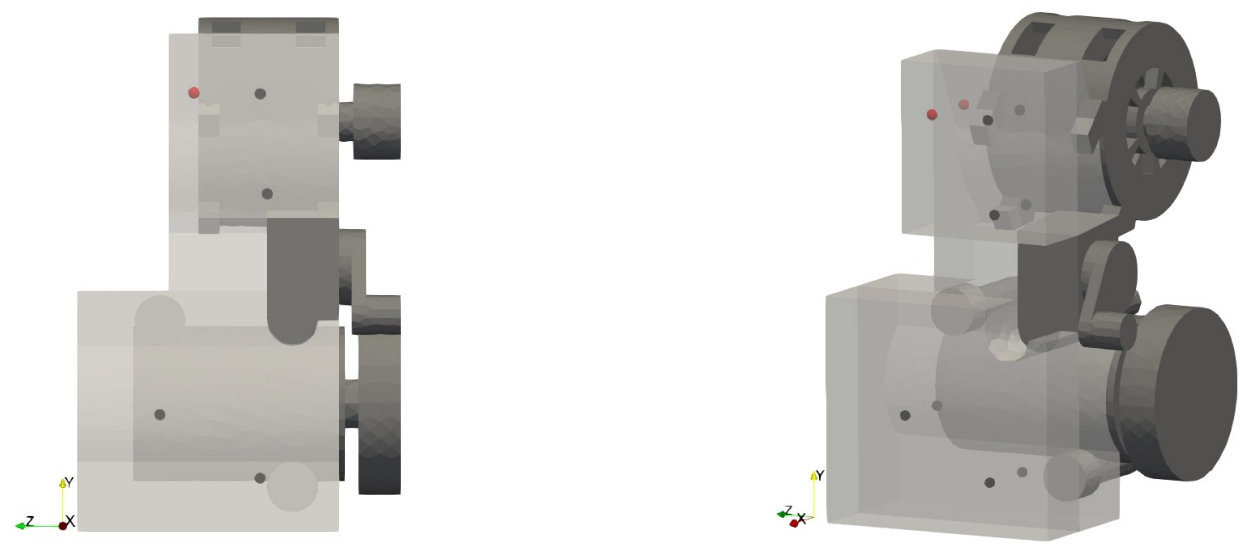

Figure 22: Fifth bolt (in red) placed by the topological derivative
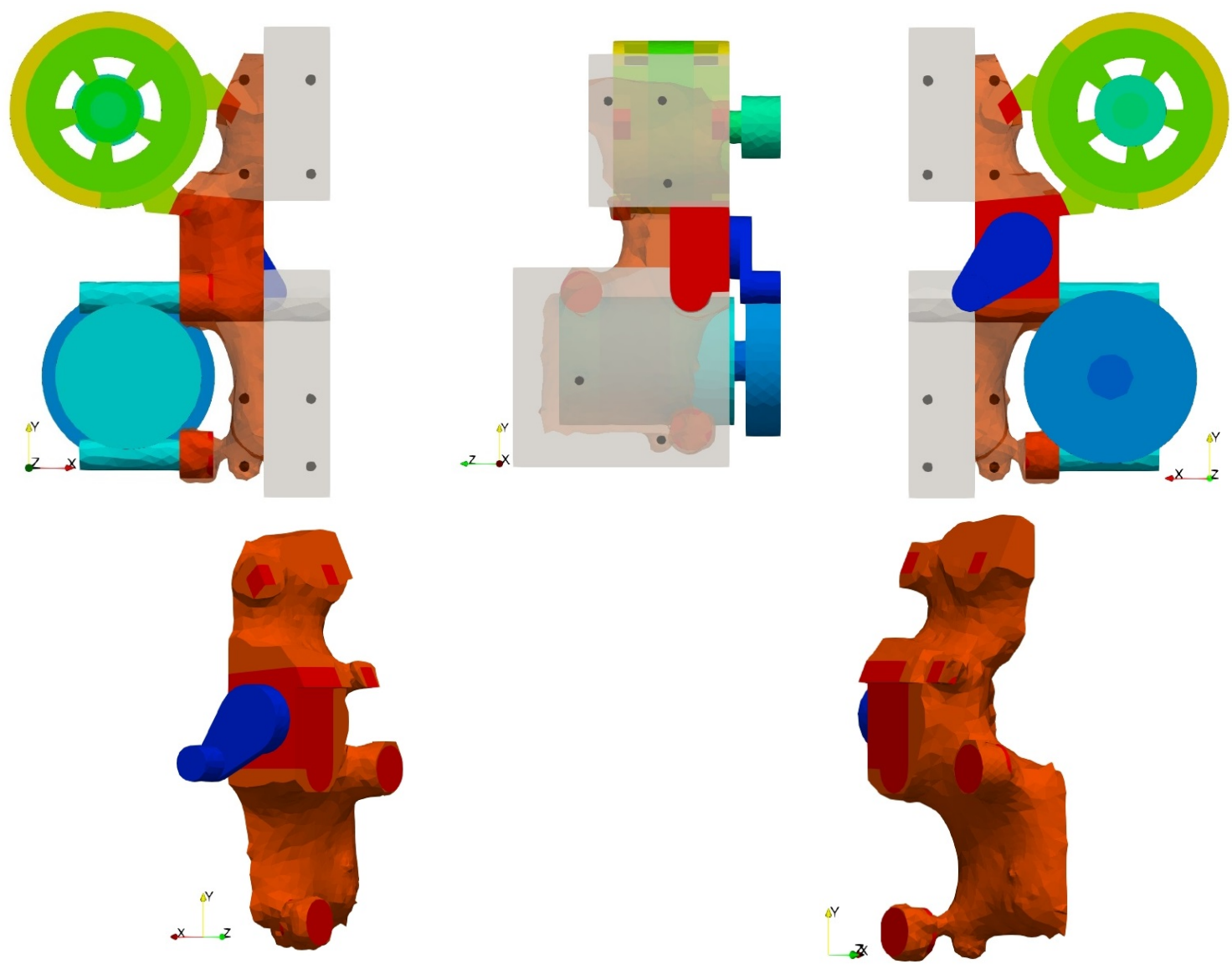

Figure 23: Final assembly after coupled optimization of structure and locations (5 bolts) 

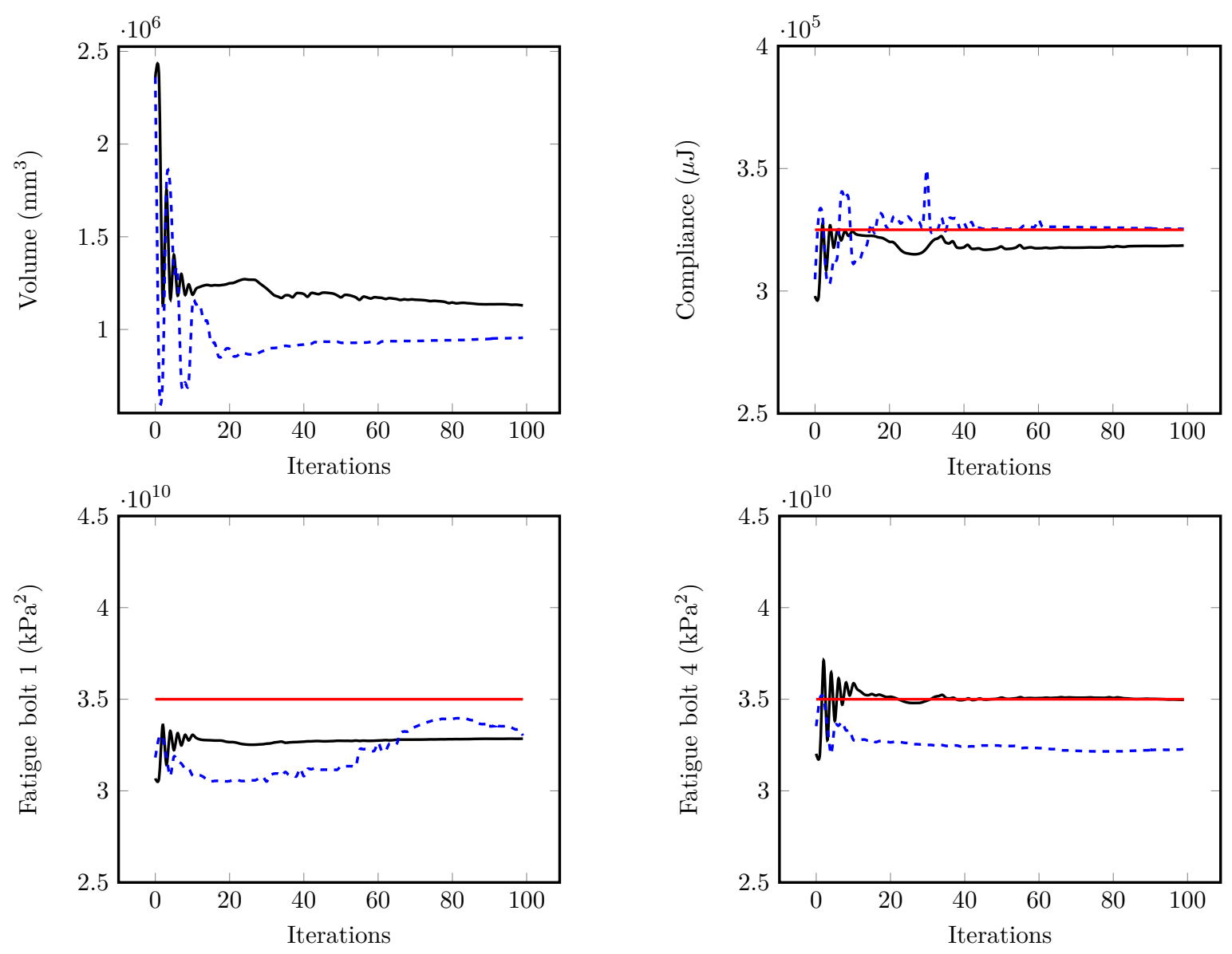

Figure 24: Convergence history of the volume, the compliance and the fatigue of bolts 1 and 4 for the problem with 7 fixed bolts (in black solid line) and with 5 bolts (in blue dashed line) 


\section{Use of six bolts}

Based on the optimal assembly with five bolts, we apply again the topological derivative to place a sixth both in order to decrease the compliance. Negative iso-values of the topological derivative are given in Fig. 25. The sixth bolt is then placed in the lower left corner of the bracket (see Fig. 26). The new compliance is $C(\Omega)=314000 \mu \mathrm{J}$. All bolts mechanical constraints are in the feasible design.

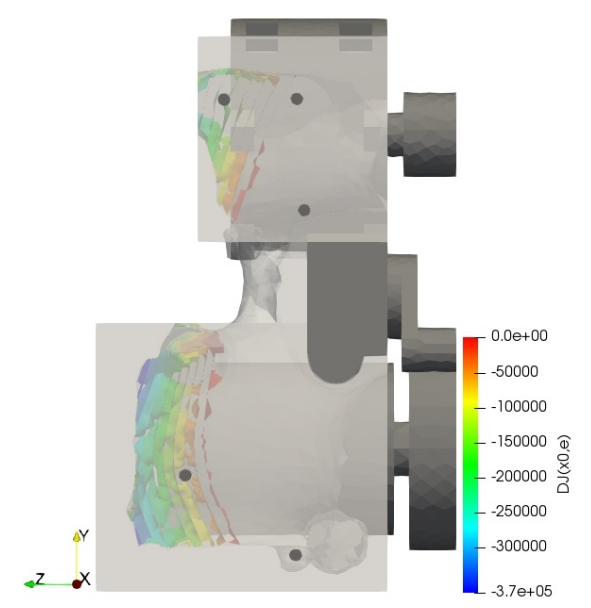

Figure 25: Iso-values of the topological derivative on the assembly with the optimal bracket for five bolts

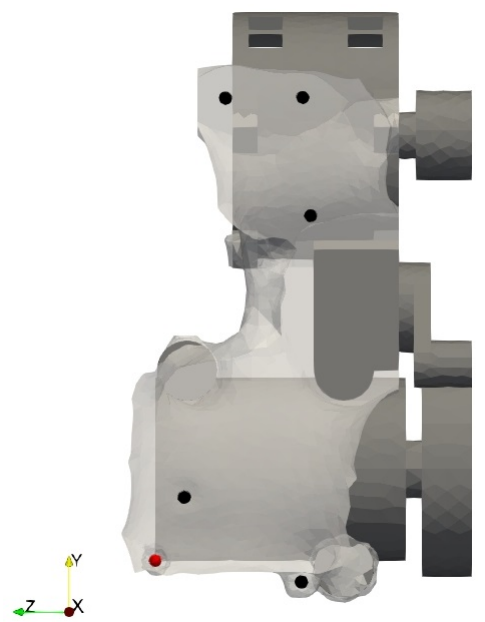

Figure 26: Sixth bolt (in red) placed by the topological derivative

We solve the problem (46) to optimize the structure of the bracket and the locations of six bolts. Final structure and bolts location are given in Fig. 27. Once again, the compliance threshold is reached and all bolts mechanical constraints are respected. Bolts location barely changed with respectively a minimum and a maximum displacement amplitude of $0.04 \mathrm{~mm}$ for bolt 2 and $0.72 \mathrm{~mm}$ for bolt 1 . The shape of the bracket does not change a lot. The add of the sixth bolt smooths curves and corners. The bracket has a final volume of $V(\Omega)=8.57 \times 10^{5}$ $\mathrm{mm}^{3}$, corresponding to a reduction of $24.14 \%$ compared to the reference result with seven fixed bolts.

Table 1 summarizes volumes of the bracket and optimization constraints according to the number of bolts and design variables. Thanks to these data, technico-economic trade-off between unit cost (the mass) and investment (the number of bolts) may be carried out.
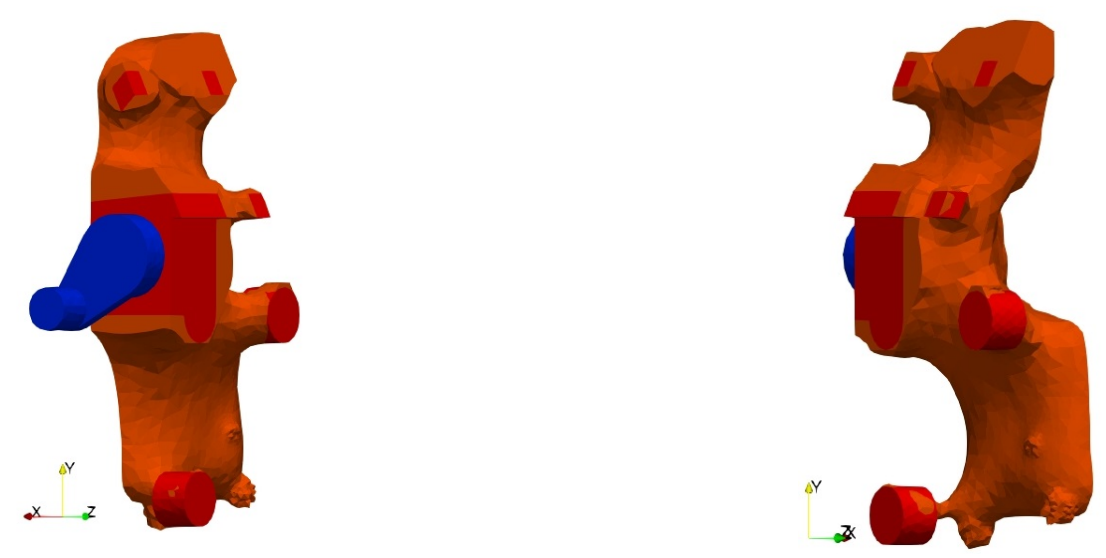

Figure 27: Final assembly for volume minimization of the bracket under compliance and bolts mechanical constraints with six bolts 


\begin{tabular}{|c|c|c|c|c|}
\hline Bolts & Design variables & Volume $\left(\mathrm{mm}^{3}\right)$ & Compliance $(\mu \mathrm{J})$ & Bolts mechanical constraints \\
\hline 7 & Structure & $1.13 \times 10^{6}$ & 319000 & All respected - 1 saturated \\
6 & Structure + Locations & $8.57 \times 10^{5}$ & 325000 & All respected \\
5 & Structure + Locations & $9.56 \times 10^{5}$ & 325000 & All respected \\
\hline
\end{tabular}

Table 1: Summary of volumes and constraints versus number of bolts and design variables

\section{Conclusion and perspectives}

In this work, we introduced a new optimization approach for assembled systems where we simultaneously optimize the shape and topology of an individual part of the system and the location and number of connecting bolts. Compared to a classical topology optimization with fixed bolts, the shape and bolts coupled optimization delivers a lighter structure and is able to reduce the number of required bolts while keeping equivalent mechanical performances. The main new ingredients are an idealized bolt model and a notion of topological derivative to decide where to introduce a new bolt. Another feature of our bolt model is the intrinsic definition of specific mechanical constraints which can be taken into account in the optimization process, ensuring maximal exploitation of the final optimized assembly by designers.

There are many perspectives and generalizations of our work. The orientation, the size and the prestressed state of the bolts could be optimized too. Additional tools are needed to suppress a useless bolt or to merge two bolts getting too close into a unique larger one. Other objective functions or mechanical constraints for which shape derivatives are available (see e.g. [22]) could be optimized, particularly the contact pressure. Different models could be accommodated in our setting, like free or forced vibrations.

\section{Acknowledgements}

The research work of L. Rakotondrainibe has been supported by the Association Nationale de la Recherche et de la Technologie (ANRT). It was carried out in the framework of a CIFRE thesis between Groupe RENAULT, Technocentre and Centre de Mathématiques Appliquées at Ecole Polytechnique (CMAP). The research work of J. Desai has been carried out in the framework of project TOP, at IRT SystemX, Paris-Saclay, France, and therefore supported by "Programme d'Investissements d'Avenir".

\section{References}

[1] NX Nastran User's Guide. Siemens PLM Software, United States, 2014.

[2] G Allaire. Conception optimale de structures. Springer-Verlag, Berlin, Mathématiques et Applications 58, 2007.

[3] G Allaire, F Jouve, and A M Toader. Structural optimization using sensitivity analysis and a level-set method. J. Comp. Phys., 194(1):363-393, 2004.

[4] E Aydin, M Dutkiewicz, B Öztürk, and M Sonmez. Optimization of elastic spring supports for cantilever beams. Structural and Multidisciplinary Optimization, 62:55-81, 2020.

[5] M Berot. Modélisation simplifiée d'assemblages par éléments équivalents. PhD thesis, École Nationale Supérieure des Mines de Paris, 2009. 
[6] J Bickford. An introduction to the design and behavior of bolted joints, volume 97 of Mechanical Engineering. Marcel Dekker, New York, 3 edition, 1995.

[7] T Buhl. Simultaneous topology optimization of structure and supports. Struct. Multidiscip. Optim., 23:336-346, 2002.

[8] J Céa, S Garreau, P Guillaume, and M Masmoudi. The shape and topological optimizations connection. Comput. Methods Appl. Mech. Engrg., 188:713-726, 2000.

[9] J A Collins, H R Busby, and G H Staab. Mechanical Design of Machine Elements and Machines. Wiley, 2009.

[10] C Dapogny, C Dobrzynski, and P Frey. Three-dimensional adaptive domain remeshing, implicit domain meshing, and applications to free and moving boundary problems. Journal of computational physics, 262:358-378, 2014.

[11] C Eck, J Jarusek, and M Krbec. Unilateral Contact Problems: Variational Methods and Existence Theorems. Chapman \& Hall/CRC Pure and Applied Mathematics. CRC Press, 2005.

[12] H Eschenauer, V Kobelev, and A Schumacher. Bubble method for topology and shape optimization of structures. Structural Optimization, 8:42-51, 1994.

[13] R Grzejda. Modelling bolted joints using a simplified model. Journal of Mechanical and Transport Engineering, 69(1):23-37, 2017.

[14] J Guillot. Assemblages par éléments filetés, volume B 5560. Techniques de l'Ingénieur, 1989.

[15] J Guillot. Modélisation et calcul des assemblages visées. Généralités, volume BM 5560. Techniques de l'Ingénieur, 1989.

[16] J Hadamard. Mémoire sur le problème d'analyse relatif à l'équilibre des plaques élastiques encastrées. Bull. Soc. Math., France, 1907.

[17] J Hou, J Zhu, J Wang, and W Zhang. Topology optimization of multi-fasteners jointed structure considering fatigue constraints. Int. J. Simul. Multidisci. Des. Optim., 9, A4, 2018.

[18] T Jiang and M Chirehdast. A systems approach to structural topology optimization: Designing optimal connections. ASME. J. Mech.- Des., 119(1):40-47, 1997.

[19] M Kerguignas and G Caignaert. Résistance des matériaux. DUNOD Université, 4ème ed., Paris, 1977.

[20] D Kinderlehrer and G Stampacchia. An introduction to variational inequalities and their applications, volume 31 of Classics in Applied Mathematics. SIAM, 1980.

[21] Q Li, G P Steven, and Y M Xie. Evolutionary structural optimization for connection topology design of multi-component systems. Engineering Computations, 18(3/4):460-479, 2001.

[22] A Maury, G Allaire, and F Jouve. Shape optimisation with the level set method for contact problems in linearised elasticity. SMAI-Journal of computational mathematics, 3:249-292, 2017.

[23] F Mignot. Contrôle dans les inéquations variationelles elliptiques. Journal of Functional Analysis, 22(2):130-185, 1976.

[24] J Montgomery. Methods for modeling bolts in the bolted joint. ANSYS World Users Conference, 2002.

[25] A A Novotny and J Sokołowski. Topological derivatives in shape optimization. Springer, Heidelberg, 2013. 
[26] A Oinonen, P Tanskanen, T Björk, and G Marquis. Pattern optimization of eccentrically loaded multi-fastener joints. Struct Multidisc Optim, 40:597-609, 2010.

[27] S J Osher and R Fedkiw. Level set methods and dynamic implicit surfaces, volume 153. Springer Science \& Business Media, Applied Mathematical Sciences, 2006.

[28] S J Osher and J A Sethian. Fronts propagating with curvature-dependent speed: Algorithms based on Hamilton-Jacobi formulations. J. Comput. Phys., 79:12-49, 1988.

[29] O Pironneau. Optimal shape design for elliptic systems. Springer-Verlag, New-York, 1984.

[30] L Rakotondrainibe. Optimisation topologique des liaisons dans les systèmes mécaniques. $\mathrm{PhD}$ thesis, Institut Polytechnique de Paris, 2020. https://tel.archives-ouvertes.fr/tel-03106332.

[31] L Rakotondrainibe, G Allaire, and P Orval. Topology optimization of connections in mechanical systems. Structural and Multidisciplinary Optimization, 61:2253-2269, 2020.

[32] L Rakotondrainibe, G Allaire, and P Orval. Topological sensitivity analysis with respect to a small idealized bolt. submitted to Engineering Computations, HAL preprint: hal-03157844, 2021.

[33] A-B Ryberg and Nilsson L. Spot weld reduction methods for automotive structures. Structural and Multidisciplinary Optimization, 53:923-934, 2016.

[34] J A Sethian. Level set methods and fast marching methods: evolving interfaces in computational geometry, fluid mechanics, computer vision, and materials science, volume 3. Cambridge university press, 1999.

[35] G Sines. Failure of materials under combines repeated stresses with superimposed static stresses. Technical report, NACA, 1955. Tech. Note, 3495.

[36] M Smith. ABAQUS/Standard User's Manual, Version 6.9. Dassault Systèmes Simulia Corp, United States, 2009.

[37] J Sokołowski and J P Zolesio. Introduction to shape optimization. Springer-Verlag, Berlin, 1992.

[38] J Sokołowski and A Żochowski. On the topological derivative in shape optimization. SIAM Journal on Control and Optimization, 37:1251-1272, 1999.

[39] J Sokołowski and A Żochowski. Topological derivatives of shape functionals for elasticity systems. Mechanics of Structures and Machines, 29:333-351, 2001.

[40] J H Son and B M Kwak. Optimization of boundary conditions for maximum fundamental frequency of vibrating structures. AIAA journal, 31(12):2351-2357, 1993.

[41] B P Wang and J L Chen. Application of genetic algorithm for the support location optimization of beams. Comput. Struct, 58:797-800, 1996.

[42] D Wang. Structural optimization with evolutionary shift method. PhD thesis, Department of Aircraft Manufacturing Engineering, Northwestern Polytechnical University, 2003.

[43] D Wang, J S Jiang, and W H Zhang. Optimization of support positions to maximize the fundamental frequency of structures. Int. J. Numer. Methods Eng., 61:1584-1602, 2004.

[44] M Y Wang, X Wang, and D Guo. A level set method for structural topology optimization. Comput. Methods Appl. Mech. Engrg., 192:227-246, 2003. 
[45] Q Xia, M Y Wang, and T Shi. A level set method for shape and topology optimization of both structure and support of continuum structures. Comput. Methods Appl. Mech. Engrg., 272:340-353, 2014.

[46] S Yang, L Yan, and C Qi. An adaptive multi-step varying-domain topology optimization method for spot weld design of automotive structures. Structural and Multidisciplinary Optimization, 59:291-310, 2019.

[47] E Zahavi. The Finite Element Method in Machine Design. Prentice-Hall, 1992.

[48] J H Zhu, H H Gao, Zhang W H, and Y Zhou. A multi-point constraints based integrated layout and topology optimization design of multi-component systems. Structural and Multidisciplinary Optimization, 51(2):397-407, 2015.

[49] J H Zhu, W J Guo, W H Zhang, and T Liu. Integrated layout and topology optimization design of multi-frame and multi-component fuselage structure systems. Structural and Multidisciplinary Optimization, 56(1):21-45, 2017.

[50] J H Zhu and W H Zhang. Maximization of structure natural frequency with optimal support layout. Structural and Multidisciplinary Optimization, 31:462-469, 2006.

[51] J H Zhu and W H Zhang. Integrated layout design of supports and structures. Computer Methods in Applied Mechanics and Engineering, 199(9-12):557-569, 2010.

[52] B Żylinski and R Buczkowski. Analysis of bolt joint using the finite element method. Archive of Mechanical Engineering, 57(3):275-292, 2010. 